\title{
ASYMPTOTIC BEHAVIOR OF MEROMORPHIC FUNCTIONS WITH EXTREMAL SPREAD \\ I
}

\author{
ALBERT EDREI and WOLFGANG H. J. FUCHS
}

Introduction. The notion of spread was introduced and investigated [2], [3] by one of us, who also conjectured the spread relation stated below as inequality (5).

This relation has now been proved by A. Baernstein II [1] whose remarkable analysis rests on the introduction of a new function $T^{*}(z)(z=$ $\left.r e^{i \theta}\right)$, closely related to Nevanlinna's characteristic $T(r, f)=T(r)$.

The importance of $T^{*}(z)$ appears sufficient to warrant further study. In this note we examine the behavior of $T^{*}(z)$ under certain assumptions which we characterize by the phrase "extremal spread".

All our results are expressed in terms of the classical definitions and notations of Nevanlinna's theory of meromorphic functions. They will be taken for granted.

Throughout the paper, $f(z)$ denotes a meromorphic function of lower order $\mu(0<\mu<+\infty)$ having deficient poles:

$$
\delta(\infty, f)>0 \text {. }
$$

Let

$$
\eta(r) \rightarrow 0 \quad(r \rightarrow \infty),
$$

(it is not assumed that $\eta(r) \geqq 0$ ) and introduce the set of arguments

$$
E(r, \infty)=E(r)=\left\{\theta: \log \left|f\left(r e^{i \theta}\right)\right| \geqq \eta(r) T(r)\right\} .
$$

The measure of the measurable set $E(r)$ will be denoted by $|E(r)|$.

We next focus our attention on $|E(r)|$ as $r \rightarrow \infty$ by values of a suitable sequence

The research of the first author was supported by NSF grant GP 33175. The second author gratefully acknowledges support by NSF grant GP 28251, by a Fulbright-Hayes Fellowship, and also the hospitality of Imperial College, London, and of the Mathematical Institute of the E.T.H., Zürich. 


$$
r_{1}, r_{2}, r_{3}, \ldots, r_{m}, \ldots \quad\left(1<r_{1}<r_{2}<r_{3}<r_{4}<\ldots ; r_{m} \rightarrow \infty\right) .
$$

We always take $\left\{r_{m}\right\}_{m}$ to be a sequence of Pólya peaks of order $\mu$ of $T(r)$. The exact definition of these peaks, given in $\S 1$, is not needed at this stage and the meaning of Theorems 1 and 2 is not lost if we simply assume that (3) is a "suitable" sequence (which always exists).

We say that

$$
\liminf _{m \rightarrow \infty}\left|E\left(r_{m}\right)\right|=\sigma(\infty, f),
$$

is the spread of $\infty$, at the sequence of peaks $\left\{r_{m}\right\}_{m}$.

It is unnecessary to explicitly mention, in the terminology, the dependence of $\sigma(\infty, f)$ on the function $\eta(r)$.

This simplified terminology does lead to some difficulties. For instance, as $r \rightarrow \infty$,

$$
\left|\left\{\theta: \log \left|\exp \left(r e^{i \theta}\right)+2\right| \geqq 0\right\}\right| \rightarrow 2 \pi,
$$

whereas

$$
\left|\left\{\theta: \log \left|\exp \left(r e^{i \theta}\right)+2\right| \geqq \log r\right\}\right| \rightarrow \pi .
$$

If one wishes to obtain a definition of the spread which is independent of $\eta(r)$, one must, following Baernstein, write

$$
\sigma(\infty, f ; \eta(r))
$$

instead of the right-hand side of (4), and then define

$$
\sigma(\infty, f)=\inf _{\eta(r)} \sigma(\infty, f ; \eta(r))
$$

where the infimum is taken over all real functions $\eta(r)$ such that $\eta(r) \rightarrow 0$ $(r \rightarrow \infty)$. It is sufficient, in this first part of our investigation, to adopt the simplified terminology and notation and to assume that the spread $\sigma(\infty, f)$ under consideration is derived from some specific, admissible choice of $\eta(r)$.

The spread relation proved by Baernstein asserts that

$$
\sigma(\infty, f) \geqq \min \left\{\frac{4}{\mu} \sin ^{-1} \sqrt{\frac{\delta(\infty, f)}{2}}, 2 \pi\right\} .
$$

Put

$$
2 \beta=\frac{4}{\mu} \sin ^{-1} \sqrt{\frac{\delta(\infty, f)}{2}} \quad\left(0<\beta \leqq \frac{\pi}{2 \mu}\right),
$$

which it is convenient to rewrite as

$$
\delta(\infty, f)=1-\cos \beta \mu .
$$


If $\mu>1 / 2$, then

$$
0<\beta<\pi
$$

and (5) reduces to

$$
\sigma(\infty, f) \geqq 2 \beta .
$$

If $0<\mu \leqq 1 / 2$, we make the additional assumption

$$
\delta(\infty, f)^{-}<1-\cos \pi \mu,
$$

so that (8) still holds and (9) is again a consequence of (5). We do not need to assume the validity of the spread relation. [We show $(\S 8)$ that it is contained in some of our inequalities.]

The aim of the present paper is to determine, in some sense, all the meromorphic functions characterized by the following

Hy p otheses ES. Let $f(z)$ be a meromorphic function of lower order $\mu$

$$
0<\mu<+\infty \text {, }
$$

and let (3) be a sequence of Pólya peaks of order $\mu$ of $T(r)=T(r, f)$.

Assume that

(i) the poles of $f$ are deficient and, if $0<\mu \leqq 1 / 2$, assume in addition that (10) holds;

(ii) the sequence $\left\{r_{m}\right\}_{m}$, of Pólya peaks of order $\mu$, is such that (4) and (5) can be replaced by

$$
\beta_{m}=\frac{1}{2}\left|E\left(r_{m}\right)\right|, \quad \beta_{m} \rightarrow \beta,
$$

where $\beta$ is given by (6).

The meaning of $r_{m}$ and of the associated quantity $\beta_{m}$ remains unchanged throughout the paper; all our assumptions and assertions refer to some specific sequence (3).

Extremal spread. If $f(z)$ satisfies the hypotheses ES, we say that it has extremal spread of $\infty$, or simply "extremal spread".

We prove

Theor em 1. Let $f(z)$ be meromorphic (or entire), of lower order $\mu(0<\mu<+\infty)$ and let $f(z)$ have extremal spread of $\infty$.

Consider the intervals

$$
I_{m}(s)=\left\{r: e^{-s} r_{m}<r \leqq e^{s} r_{m}\right\} \quad(s>0, m=1,2,3, \ldots)
$$

and let $r \rightarrow \infty$ by values restricted to the set

$$
\Lambda(s)=\bigcup_{m=1}^{\infty} I_{m}(s) .
$$


Then, for every $s>0$, we have

$$
\begin{aligned}
& \frac{T(r) / r^{\mu}}{T\left(r_{m}\right) / r_{m}{ }^{\mu}} \rightarrow 1 \quad\left(r \in I_{m}(s)\right), \\
& \frac{N(r, f)}{T(r)} \rightarrow \cos \beta \mu, \\
& \frac{n(r, f)}{T(r)} \rightarrow \mu \cos \beta \mu .
\end{aligned}
$$

It is useful to note the following

Restatement of (15). Let $T(r)$ be positive and nondecreasing and let (15) hold for every $s>0$.

It is then possible to find three positive sequences $\left\{R_{m}^{\prime}\right\},\left\{R_{m}^{\prime \prime}\right\},\left\{\tilde{\varepsilon}_{m}\right\}$ such that, as $m \rightarrow+\infty$,

$$
R_{m}^{\prime} \rightarrow+\infty, \quad r_{m} / R_{m}^{\prime} \rightarrow+\infty, \quad R_{m}^{\prime \prime} / r_{m} \rightarrow+\infty, \quad \tilde{\varepsilon}_{m} \rightarrow 0,
$$

and such that

$$
R_{m}^{\prime} \leqq t \leqq R_{m}^{\prime \prime} \quad\left(m>m_{0}\right)
$$

imply

$$
\left(t / r_{m}\right)^{\mu}\left(1+\tilde{\varepsilon}_{m}\right)^{-1} \leqq T(t) / T\left(r_{m}\right) \leqq\left(t / r_{m}\right)^{\mu}\left(1+\tilde{\varepsilon}_{m}\right) .
$$

A proof of these remarks will be found in [3; p. 323, Lemma 4]; a less elementary approach (using Egoroff's theorem) shows that the assumption that $T(r)$ is nondecreasing may be omitted [7, p. 52].

Baernstein's proof of the spread relation (5) is based on the properties of the function, introduced by him,

$$
T^{*}\left(r e^{i \theta}\right)=m^{*}\left(r e^{i \theta}\right)+N(r, f),
$$

where

$$
m *\left(r e^{i \theta}\right)=\sup _{|E|=2 \theta} \frac{1}{2 \pi} \int_{E} \log \left|f\left(r e^{i \varphi}\right)\right| d \varphi ;
$$

the "sup" is taken over all measurable sets of measure $|E|=2 \theta$.

Baernstein showed that $T^{*}\left(r e^{i \theta}\right)$ is a subharmonic function in $0<r<\infty, 0<\theta<\pi$.

Our study of $T^{*}\left(r e^{i \theta}\right)$, under the hypotheses ES, yields, in addition to Theorem 1, the following

$\mathrm{Th}$ e o $\mathrm{rem}$ 2. Let the assumptions and notations of Theorem 1 be unchanged. Then, given $s>0$, there exists a sequence $\left\{\eta_{m}\right\}_{m}, \eta_{m} \rightarrow 0$, independent of $r$ and $\theta$, such that 


$$
\left|T^{*}\left(r e^{i \theta}\right)-T(r) \cos \mu(\beta-\theta)\right|<\eta_{m} T(r)
$$

provided

$$
r \in I_{m}(s), \quad 0 \leqq \theta \leqq \beta .
$$

It is possible to prove a good deal more than asserted in Theorems 1 and 2. For values of $z$ which lie in the annuli

$$
|z| \in I_{m}(s) \quad(m=1,2,3, \ldots),
$$

we can satisfactorily determine the asymptotic behavior of $\log |f(z)|$ and of the arguments of almost all the zeros and all the poles in these annuli.

This detailed study would lengthen inordinately the present paper. We shall therefore be content to prove the following Theorem 3 and expect to return, on some future occasion, to the more exhaustive study of $\log |f(z)|$ and of the angular distribution of all the zeros of $f(z)$. [The behavior of the poles is already described in the following result.]

Theorem 3. Let the assumptions and notations of Theorem 1 be unchanged.

It is then yossible to find real sequences $\left\{u_{m}\right\}_{m}$, and $\left\{\omega_{m}\right\}_{m}$ satisfying all the following conditions

$$
\begin{aligned}
& \text { I. } u_{m} \sim r_{m} \quad(m \rightarrow \infty) . \\
& \text { II. } f\left(u_{m} e^{i \theta}\right) \neq \infty \quad(0 \leqq \theta<2 \pi) . \\
& \text { III. }\left|f\left(u_{m} e^{i \omega_{m}}\right)\right|=M\left(u_{m}\right)=\max _{|z|=u_{m}}|f(z)| . \\
& \text { IV. } \lim _{m \rightarrow \infty} \frac{\log M\left(u_{m}\right)}{T\left(u_{m}\right)}=\pi \mu \sin \mu \beta .
\end{aligned}
$$

V. Let $s>0$ and $\eta(0<\eta<\beta)$ be given. Denote by $\mu_{m}$ the number of poles of $f(z)$ in the sector

$$
\left\{r e^{i \theta}: u_{m} e^{-s}<r \leqq u_{m} e^{s}, \eta \leqq\left|\theta-\omega_{m}\right| \leqq \pi\right\}
$$

and by $x_{m}$ the number of zeros $f(z)$ in the sector

$$
\left\{r e^{i \theta}: u_{m} e^{-s}<r \leqq u_{m} e^{s},\left|\theta-\omega_{m}\right| \leqq \beta-\eta\right\} .
$$

Then

$$
\mu_{m}+x_{m}=o\left(T\left(u_{m}\right)\right) \quad(m \rightarrow \infty) .
$$

An inspection of our analysis immediately suggests the construction of Functions extremal for the spread relation. Let $\mu$ and $\delta$ be given

$$
0<\mu<+\infty
$$


and

$$
\begin{cases}0<\delta<1 & \left(\frac{1}{2}<\mu\right) \\ 0<\delta<1-\cos \pi \mu & \left(0<\mu \leqq \frac{1}{2}\right)\end{cases}
$$

Define auxiliary quantities $p, \beta>0, \alpha>0$ :

$$
p=[\mu], \quad \beta \mu=2 \sin ^{-1} \sqrt{\frac{\delta}{2}}<\frac{\pi}{2}, \quad \alpha=(2 \cos \beta \mu)^{-1 / \mu} .
$$

Consider the canonical products

$$
g(z)=\prod_{n=1}^{\infty} E\left(-\frac{z}{n^{1 / \mu}}, p\right) \quad\left(E(t, p)=(1-t) e^{t+\frac{t^{2}}{2}+\ldots+\frac{t^{p}}{p}}\right),
$$

and the meromorphic functions

$$
\begin{cases}\mathscr{K}(z)=\frac{g\left(\alpha e^{i \beta} z\right) g\left(\alpha e^{-i \beta} z\right)}{g(z)} & (\mu \neq \text { integer }), \\ \mathscr{K}(z)=\frac{g\left(\alpha e^{i \beta} z\right) g\left(\alpha e^{-i \beta} z\right)}{g(z)} e^{z z} & (\mu=\text { integer }),\end{cases}
$$

with

$$
\chi=(-1)^{p} \beta \tan \beta p+(-1)^{p-1} \log \alpha .
$$

Then, for any given $\eta(0<\eta<\min \{\beta / 2, \pi-\beta\})$, we have

I. If $\mu$ is not an integer,

$$
\begin{array}{r}
|\log | \mathscr{K}\left(r e^{i \theta}\right)|| \leqq \frac{K}{\sin (\eta / 2)}\left(1+\log ^{+} r\right) r^{p} \quad(|\theta| \leqq \pi-\beta-\eta), \\
|\log | \mathscr{K}\left(r e^{i \theta}\right)\left|-\frac{\pi r^{\mu} \sin \mu(\theta+\beta-\pi)}{\cos (\beta \mu)}\right| \leqq \frac{K}{\sin (\eta / 2)}\left(1+\stackrel{+}{+}{ }^{+} r\right) r^{p} \\
(\pi-\beta+\eta \leqq \theta \leqq \pi-\eta),
\end{array}
$$

where $K=K(\mu, \delta)>0$ depends only on the parameters $\mu$ and $\delta$.

II. If $\mu=p$ is an integer, (28) and (29) are still valid with $p$ replaced by $(p-1)$ in the right-hand sides. If we also assume

$$
r>2(1+1 / \alpha)
$$

the constants $K$ depend only on $\mu$ and $\delta$.

The evaluation of $T(r, \mathscr{K})$ is now straightforward but requires some attention since we must still let $\eta \rightarrow 0$. We find

If $\mu$ is not an integer 


$$
T(r, \mathscr{K})=\frac{r^{\mu}}{\mu \cos (\beta \mu)}+o\left(r^{\frac{p+\mu}{2}+\varepsilon}\right) \quad(r \rightarrow \infty),
$$

for every $\varepsilon>0$.

If $\mu$ is an integer, the 0 -term is to replaced by $O\left(r^{p-\frac{1}{2}+\varepsilon}\right)$.

The obvious relation

$$
N(r, \mathscr{K})=\frac{r^{\mu}}{\mu}+O(\log r) \quad(r \rightarrow \infty),
$$

(30) and (27) imply

$$
1-\delta(\infty, f)=\cos (\beta \mu)=1-\delta .
$$

[Integral values of $\mu$ play no exceptional role.]

The value of the spread is deduced from (28), (29) and their analogues for integral orders.

Taking, in (2),

$$
\eta(r) T(r)=r^{\mu} / \log r \quad(r>e),
$$

we find

$$
\sigma(\infty, \mathscr{K})=\lim _{r \rightarrow \infty}|E(r)|=2 \beta=\frac{4}{\mu} \sin ^{-1} \sqrt{\frac{\delta(\infty, \mathscr{K})}{2}} .
$$

Hence:

For any preassigned values of $\mu(0<\mu<+\infty)$ and $\delta(\infty, f)$, such that

$$
\begin{cases}0<\delta(\infty, f)<1 & \left(\frac{1}{2}<\mu\right), \\ 0<\delta(\infty, f)<1-\cos \pi \mu & \left(0<\mu \leqq \frac{1}{2}\right),\end{cases}
$$

there exists some $f=\mathscr{K}$ for which equality holds in the spread relation (5).

In the limiting case $\delta(\infty, f)=0$ there is nothing to prove. For $\delta(\infty, f)=1$ and $\mu>1 / 2$, it is easily seen that the functions $f=\mathscr{G}(z)$ :

$$
\begin{cases}\mathscr{G}(z)=g\left(z e^{i \frac{\pi}{2 \mu}}\right) g\left(z e^{-i \frac{\pi}{2 \mu}}\right) & \left(\frac{1}{2}<\mu \neq \text { integer }\right), \\ \mathscr{G}(z)=g\left(z e^{i \frac{\pi}{2 \mu}}\right) g\left(z e^{-i \frac{\pi}{2 \mu}}\right) \exp \left((-1)^{\mu} \frac{\pi}{\mu} z^{\mu}\right) & (0<\mu=p=\text { integer }),\end{cases}
$$

have extremal spread of $\infty$.

One final remark concerns the following result, proved in $\S 5$.

Continuity Lemma. If $f(z)$ is meromorphic in $|t| \leqq R$, $f(0)=1$, and if

$$
\begin{aligned}
& 0<t \leqq \frac{1}{4} R, \quad 0<t^{\prime} \leqq \frac{1}{4} R \\
& (1+\sigma)^{-1} \leqq t / t^{\prime} \leqq(1+\sigma) \quad(\sigma \geqq 0),
\end{aligned}
$$


then for any measurable subset $J$ of the reals, modulo $2 \pi$,

$$
\left|m(t, f ; J)-m\left(t^{\prime}, f ; J\right)\right| \leqq A \sigma\left(1+\log \frac{1}{\sigma}\right) T(R, f),
$$

where $A$ is an absolute constant and

$$
m(t, f ; J)=\frac{1}{2 \pi} \int_{J}^{++} \log \left|f\left(r e^{i \theta}\right)\right| d \theta .
$$

Similarly

$\left|\frac{1}{2 \pi} \int_{J} \log \right| f\left(t e^{i \theta}\right)\left|d \theta-\frac{1}{2 \pi} \int_{J} \log \right| f\left(t^{\prime} e^{i \theta}\right)|d \theta| \leqq A \sigma\left(1+\log \frac{1}{\sigma}\right) T(R, f)$.

If the normalization at the origin is omitted, (31) and (32) remain true provided:

(i) $f(z)$ is nonconstant;

(ii) $R>R_{0}$, where $R_{0}=R_{0}(f)$ is a suitable bound depending only on $f$.

The preceding lemma, which is needed here, is quoted and used by Baernstein $\left[1 ;\right.$ p. 422] to establish some properties of $T^{*}$. Since a proof of the continuity lemma has never been published, it may be of interest to point out that this gap is now filled.

1. Definition of Pólya peaks and notational conventions. In the following definition $T(r)$ denotes a positive function of the positive variable, not necessarily the characteristic of some meromorphic function.

Definition of $\mathrm{P}$ óly a peaks. Let the function $T(r)$ be defined for $r \geqq r_{0}>0$, and let it be positive, continuous and nondecreasing. We say that $\left\{r_{m}\right\}_{m=1}^{\infty}$ is a sequence of Pólya peaks of order $\varrho$ of $T(r)$ if there exist positive sequences

$$
\left\{r_{m}^{\prime}\right\},\left\{r_{m}^{\prime \prime}\right\},\left\{\varepsilon_{m}\right\}
$$

such that, as $m \rightarrow \infty$,

$$
\dot{r}_{m}^{\prime} \rightarrow \infty, \quad \frac{r_{m}}{r_{m}^{\prime}} \rightarrow \infty, \quad \frac{r_{m}^{\prime \prime}}{r_{m}} \rightarrow \infty, \quad \varepsilon_{m} \rightarrow 0,
$$

and such that for

$$
r_{m}^{\prime} \leqq r \leqq r_{m}^{\prime \prime},
$$

we have 


$$
T(r) \leqq\left(1+\varepsilon_{m}\right)\left(\frac{r}{r_{m}}\right)^{e} T\left(r_{m}\right)=V(r) .
$$

In our proofs there will be frequent references to the associated sequences (1.1) and to the peak inequality (1.3). We also find it convenient to introduce peak intervals of two kinds:

$$
I_{m}=\left[r_{m}^{\prime}, r_{m}^{\prime \prime}\right], \quad I_{m}(s)=\left[e^{-s} r_{m}, e^{s} r_{m}\right] \quad(s>0),
$$

and to consider their unions

$$
\Lambda=\bigcup_{m=1}^{\infty} I_{m}, \quad \Lambda(s)=\bigcup_{m=1}^{\infty} I_{m}(s) .
$$

Note that the associated sequences are by no means uniquely defined by $\left\{r_{m}\right\}$ and the conditions (1.2).

We always make the additional assumptions

$$
r_{m}^{\prime}<r_{m}<r_{m}^{\prime \prime}<r_{m+1}^{\prime} \quad(m=1,2,3, \ldots) .
$$

The nature of our results is such that this is an insignificant loss of generality and offers the advantage that the function

$$
m=m(r)
$$

is uniquely defined by the condition $r \in I_{m}$.

Most of our limiting processes involve

$$
r \rightarrow \infty, \quad r \in \Lambda(s) \quad(0<s),
$$

and lead to relations such as (15) of our Theorem 1. It is essential for the proper understanding of this typical formula to observe that $m=m(r)$ is the function (1.7). If $r$ is large enough, (1.2) and (1.6) determine without ambiguity the value of $m$ under consideration.

The decomposition

$$
z=r e^{i \theta} \quad(r \geqq 0, \theta \text { real })
$$

is used systematically.

The argument $\theta$ is only defined $\bmod 2 \pi$ and sets of arguments, whenever they appear, are restricted by this convention.

Many of our asymptotic formulae hold uniformly as certain parameters, say $t$ and $\theta$, are restricted by relations such as

$$
t \in \Lambda \quad(\text { or } t \in \Lambda(s)), \quad \theta_{1}<\theta<\theta_{2} \text {. }
$$

In order to state or stress this uniformity we consider functions $\eta(t)$ or sequences $\left\{\eta_{m}\right\}_{m}$ such that 


$$
\eta(t) \rightarrow 0 \quad(t \rightarrow \infty), \quad \eta_{m} \rightarrow 0 \quad(m \rightarrow \infty) .
$$

We add some clarity to these notations by restricting them as follows.

Assuming that conditions such as (1.9) are imposed

(i) we only use the symbol $\eta(t)$ to denote a function independent of $\theta$;

(ii) we only use the symbols $\left\{\eta_{m}\right\}_{m}$ to denote a sequence independent of $t$ and $\theta$.

If there are no restrictions such as (1.9), $\eta(t)$ and $\eta_{m}$ will be used with the obvious meaning stated in (1.10).

We frequently need to consider means of $\log |f|$ or $\log |f|$ taken on certain measurable subsets of $(-\pi, \pi]$. If $J$ is such a subset we use the notations

$$
\begin{aligned}
& m(r, f ; J)=\frac{1}{2 \pi} \int_{J} \log \left|f\left(r e^{i \theta}\right)\right| d \theta, \\
& m(r, f ; J)=\frac{1}{2 \pi} \int_{J} \log \left|f\left(r e^{i \theta}\right)\right| d \theta .
\end{aligned}
$$

Positive absolute constants are denoted by $A$ and positive constants depending on one or more parameters by $K$.

Restrictions such as $r>r_{0}, m>m_{0}, \ldots$ immediately following some relation mean that the relation in question only holds for sufficiently large values of $r, m, \ldots$. It is understood that the quantities $A, K, r_{0}, m_{0}, \ldots$, the functions $\eta(t)$, and the sequences $\left\{\eta_{m}\right\}_{m}$ are not necessarily the same ones each time they occur. Whenever we wish to stress the importance of certain parameters, say $s, \varepsilon, \delta, \ldots$ on which $K, r_{0}, m_{0}, \ldots$ may depend, we write, for instance, $K(s, \varepsilon, \delta), r_{0}=r_{0}(s, \varepsilon), m_{0}=m_{0}(\delta), \ldots$.

Statements and proofs of our results will be simplified by a strict adherence to the preceding terminology and notations: from this point on we take them for granted.

\section{A modification of Nevanlinna's form of the Phragmén-Lindelöf}

principle. The following lemma assumes and asserts a little more than an analogous modification [4; p. 159] of Nevanlinna's classical version of the Phragmén - Lindelöf principle.

$\mathrm{L}$ e $\mathrm{m} \mathrm{m}$ a 2.1. Let $\varrho>0$ and let $u(z)$ be subharmonic in

$$
\mathscr{D}: l<r<R, \quad \theta_{1}<\theta<\theta_{2},
$$

where

$$
0<2 l<R, \quad \theta_{2}-\theta_{1}=\frac{\pi}{\gamma}<\frac{\pi}{\varrho} \quad\left(\gamma>\frac{1}{2}\right)
$$




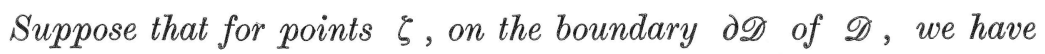

$$
\limsup _{\substack{z \rightarrow \zeta \\ z \in \mathscr{D}}} u(z) \leqq h_{1} r^{Q} \quad\left(\zeta \in \partial \mathscr{D}, \zeta=r e^{i \theta_{1}}\right),
$$$$
\limsup _{\substack{z \rightarrow \zeta \\ z \in \mathscr{D}}} u(z) \leqq h_{2} r^{\varrho} \quad\left(\zeta \in \partial \mathscr{D}, \zeta=r e^{i \theta_{2}}\right),
$$$$
\limsup _{\substack{z \rightarrow \zeta \\ z \in \mathscr{D}}} u(z) \leqq B l^{e} \quad(\zeta \in \partial \mathscr{D},|\zeta|=l)
$$

$$
\limsup _{\substack{z \rightarrow \zeta \\ z \in \mathscr{D}}} u(z) \leqq B R^{Q} \quad(\zeta \in \partial \mathscr{D},|\zeta|=R)
$$

where $h_{1}, h_{2}, B$ are real constants and $B>0$.

Put

$$
H(\theta)=h_{1} \frac{\sin \varrho\left(\theta_{2}-\theta\right)}{\sin \varrho\left(\theta_{2}-\theta_{1}\right)}+h_{2} \frac{\sin \varrho\left(\theta-\theta_{1}\right)}{\sin \varrho\left(\theta_{2}-\theta_{1}\right)} .
$$

Then, the conditions

$$
r e^{i \theta} \in \mathscr{D}, \quad l<r<\frac{1}{2} R
$$

imply

$$
u(z) \leqq r^{\varrho}\left\{H(\theta)+3\left(B+\frac{\left|h_{1}\right|+\left|h_{2}\right|}{\sin \varrho\left(\theta_{2}-\theta_{1}\right)}\right)\left[\left(\frac{l}{r}\right)^{\varrho}+\left(\frac{r}{R}\right)^{\gamma-\varrho}\right]\right\} .
$$

Proof. Consider

$$
Q(z)=-\frac{2}{\pi} \operatorname{Im}\left\{\log \left(\frac{R^{\gamma}-z^{\gamma} \exp \left(-i \gamma \theta_{1}\right)}{R^{\gamma}+z^{\gamma} \exp \left(-i \gamma \theta_{1}\right)}\right)\right\},
$$

which is the harmonic measure of the boundary arc

$$
z=\operatorname{Re}^{i \theta} \quad\left(\theta_{1}<\theta<\theta_{2}\right),
$$

with respect to the angular region

$$
\mathscr{D}_{0}: 0<r<R, \quad \theta_{1}<\theta<\theta_{2} .
$$

It is readily verified that the conditions

$$
0<r<\frac{R}{2}, \quad \theta_{1}<\theta<\theta_{2}, \quad \gamma>\frac{1}{2},
$$

imply 


$$
0<Q(z)<\frac{4}{\pi\left(1-2^{-2 \gamma}\right)}\left(\frac{r}{R}\right)^{\gamma}<\frac{8}{\pi}\left(\frac{r}{R}\right)^{\gamma}
$$

The function

$$
P(z)=r^{\varrho} H(\theta)+l^{\varrho}\left(B+\frac{\left|h_{1}\right|+\left|h_{2}\right|}{\sin \varrho\left(\theta_{2}-\theta_{1}\right)}\right)+R^{\varrho}\left(B+\frac{\left|h_{1}\right|+\left|h_{2}\right|}{\sin \varrho\left(\theta_{2}-\theta_{1}\right)}\right) Q(z)
$$

is harmonic for $z \in \mathscr{D}$ and, consequently,

$$
w(z)=u(z)-P(z)
$$

is subharmonic in $\mathscr{D}$.

The study of

$$
\liminf _{\substack{z \rightarrow \zeta \\ z \in \mathscr{D}}} P(z) \quad(\zeta \in \partial \mathscr{D})
$$

is almost immediate; combined with the assumptions $(2.1)-(2.4)$ it yields

$$
\limsup _{\substack{z \rightarrow \zeta \\ z \in \mathscr{D}}} w(z) \leqq 0 \quad(\zeta \in \partial \mathscr{D}),
$$

and hence $[8 ;$ p. 155, Theorem 201]

(2.9) $u(z) \leqq r^{\varrho}\left\{H(\theta)+\left(B+\frac{\left|h_{1}\right|+\left|h_{2}\right|}{\sin \varrho\left(\theta_{2}-\theta_{1}\right)}\right)\left[\left(\frac{l}{r}\right)^{\varrho}+\left(\frac{R}{r}\right)^{\varrho} Q(z)\right]\right\}$.

In view of (2.8) we deduce (2.7) from (2.9). This completes the proof of Lemma 2.1.

3. Conditions which depress the value of $u(z)$. With additional information on the boundary values of $u(z)$ the conclusions of Lemma 2.1 can be strengthened. We prove

$\mathrm{L}$ e $\mathrm{m} \mathrm{m}$ a 3.1. Let the assumptions and notations of Lemma 2.1 be unchanged. Suppose further that there exist positive quantities $\xi, x, \sigma$ such that

$$
\limsup _{\substack{z \rightarrow t e^{i \theta_{1}} \\ z \in \mathscr{D}}} u(z) \leqq\left(h_{1}-\xi\right) t^{\varrho} \quad\left(x e^{-\sigma} \leqq t \leqq x e^{\sigma}\right)
$$

Then, if $s>0$ satisfies the conditions

$$
l<x e^{-2 s}, \quad x e^{2 s}<\frac{1}{2} R,
$$

and if 


$$
z \in \mathscr{D}, \quad e^{-2 s} x \leqq|z| \leqq e^{2 s} x
$$

we have

$$
\begin{array}{r}
u(z)<r^{\varrho}\left\{H(\theta)+3\left(B+\frac{\xi+\left|h_{1}\right|+\left|h_{2}\right|}{\sin \varrho\left(\theta_{2}-\theta_{1}\right)}\right)\left[\left(\frac{l}{r}\right)^{\varrho}+\left(\frac{r}{R}\right)^{\gamma-\varrho}\right]\right. \\
\left.-\xi K_{1} \sin \pi\left(\frac{\theta-\theta_{1}}{\theta_{2}-\theta_{1}}\right)\right\},
\end{array}
$$

with

$$
K_{1}=(8 \pi)^{-1} \exp (-4 \gamma(2 s+\sigma))\{\exp (\gamma \sigma)-1\} .
$$

$\mathrm{R}$ e $\mathrm{m}$ a $\mathrm{rk}$. With minor modifications our proof shows that (3.4) and (3.5) still hold if the assumption (3.1) is replaced by

$$
\limsup _{\substack{z \rightarrow t e^{i \theta_{2}} \\ z \in \mathscr{D}}} u(z) \leqq\left(h_{2}-\xi\right) t^{e} \quad\left(x e^{-\sigma} \leqq t \leqq x e^{\sigma}\right) .
$$

Proof. Let $g(t)$ be a continuous function of $t$ such that

$$
\begin{aligned}
g(t) & =0 \quad\left(0 \leqq t, t \notin\left[x e^{-\sigma}, x e^{\sigma}\right]\right), \\
g(t) & =\xi t^{\varrho} \quad\left(x e^{-\sigma / 2} \leqq t \leqq x e^{\sigma / 2}\right) \\
0 & \leqq g(t) \leqq \xi t^{\varrho} \quad(0 \leqq t)
\end{aligned}
$$

Consider now

$$
\begin{aligned}
v(z) & =\operatorname{Im}\left\{\frac{\gamma}{\pi} \int_{0}^{\infty} \frac{g(t) t^{\gamma-1}}{t^{\gamma}-z^{\gamma} \exp \left(-i \gamma \theta_{1}\right)} d t\right\} \\
& =\frac{\gamma}{\pi} \int_{0}^{\infty} \frac{g(t) t^{\gamma-1} r^{\gamma} \sin \gamma\left(\theta-\theta_{1}\right)}{t^{2 \gamma}-2 t^{\gamma} r^{\gamma} \cos \gamma\left(\theta-\theta_{1}\right)+r^{2 \gamma}} d t,
\end{aligned}
$$

which is obviously harmonic in the angular region

$$
\mathscr{A}: r>0, \quad \theta_{1}<\theta<\theta_{2} \text {, }
$$

and remains continuous on the sides of the angle. The evaluation of the boundary values is elementary and well known. We find

$$
\left\{\begin{array}{l}
\lim _{\substack{z \rightarrow \zeta \\
z \in \mathscr{A}}} v(z)=g(t) \quad\left(\zeta=t e^{i \theta_{1}}, t \geqq 0\right), \\
\lim _{\substack{z \rightarrow \zeta \\
z \in \mathscr{A}}} v(z)=0 \quad\left(\zeta=t e^{i \theta_{2}}, t \geqq 0\right) .
\end{array}\right.
$$


In view of (3.8) and (3.9) it is clear that

$$
\begin{aligned}
0 & \leqq v(z) \leqq \xi \frac{\gamma}{\pi} \int_{0}^{\infty} \frac{t^{\varrho+\gamma-1} r^{\gamma} \sin \gamma\left(\theta-\theta_{1}\right)}{t^{2 \gamma}-2 t^{\gamma} r^{\gamma} \cos \gamma\left(\theta-\theta_{1}\right)+r^{2 \gamma}} d t \\
& =\xi r^{\varrho} \frac{\sin \varrho\left(\theta-\theta_{1}\right)}{\sin \varrho\left(\theta_{2}-\theta_{1}\right)} \quad(z \in \mathscr{A}) .
\end{aligned}
$$

The subharmonic function

$$
u_{1}(z)=u(z)+v(z)
$$

satisfies the boundary conditions

$$
\limsup _{\substack{z \rightarrow \zeta \\ z \in \mathscr{D}}} u_{1}(z) \leqq \limsup _{\substack{z \rightarrow \zeta \\ z \in \mathscr{D}}} u(z)+g(t) \leqq h_{1} t^{e} \quad\left(\zeta=t e^{i \theta_{1}} \in \partial \mathscr{D}\right)
$$

this is an obvious consequence of (3.1), (3.10), (3.6) and (3.8).

Similarly,

(3.13) $\quad \lim \sup u_{1}(z) \leqq \lim \sup u(z) \leqq h_{2} t^{\circ} \quad\left(\zeta=t e^{i \theta_{2}} \in \partial \mathscr{D}\right)$.

$$
\underset{\substack{z \rightarrow \zeta \\ z \in \mathscr{D}}}{\substack{z \rightarrow \zeta \\ z \in \mathscr{D}}}
$$

From (2.3) and (3.11)

(3.14) $\quad \limsup _{\substack{z \rightarrow \zeta \\ z \in \mathscr{D}}} u_{1}(z) \leqq\left(B+\frac{\xi}{\sin \varrho\left(\theta_{2}-\theta_{1}\right)}\right) l^{\varrho} \quad(|\zeta|=l, \zeta \in \partial \mathscr{D})$.

Similarly (2.4) and (3.11) yield

(3.15) $\quad \limsup _{\substack{z \rightarrow \zeta \\ z \in \mathscr{D}}} u_{1}(z) \leqq\left(B+\frac{\xi}{\sin \varrho\left(\theta_{2}-\theta_{1}\right)}\right) R^{\varrho} \quad(|\zeta|=R, \zeta \in \partial \mathscr{D})$.

An inspection of (3.12), (3.13), (3.14) and (3.15) shows that Lemma 2.1 is applicable to $u_{1}(z)$ (with $B$ replaced $B+\xi /\left(\sin \varrho\left(\theta_{2}-\theta_{1}\right)\right)$. Hence, in view of (3.2) and (3.3), we deduce from (2.7)

$$
u(z) \leqq r^{\varrho}\left\{H(\theta)+3\left(B+\frac{\xi+\left|h_{1}\right|+\left|h_{2}\right|}{\sin \varrho\left(\theta_{2}-\theta_{1}\right)}\right)\left[\left(\frac{l}{r}\right)^{\varrho}+\left(\frac{r}{R}\right)^{\gamma-\varrho}\right]\right\}-v(z) .
$$

In order to complete the proof of Lemma 3.1 it is sufficient to obtain a lower bound for $v(z)$ under the restrictions (3.3).

Since $g(t) \geqq 0$, it follows from (3.7) and (3.9) that, 


$$
v(z) \geqq \frac{\xi r^{\varrho} \sin \gamma\left(\theta-\theta_{1}\right)}{\pi} \int_{x \exp (-\sigma / 2)}^{x \exp (\sigma / 2)} \frac{\gamma t^{\varrho+\gamma-1}}{r^{\varrho+\gamma}\left\{1+(t / r)^{\gamma}\right\}^{2}} d t .
$$

Remembering the definition of $\gamma$ and noting that, in the range of integration,

$$
\left\{1+(t / r)^{\gamma}\right\}^{-2}>\frac{1}{4} \exp \left\{-2 \gamma\left(2 s+\frac{1}{2} \sigma\right)\right\}
$$

we find

$$
\begin{aligned}
v(z) & \geqq \frac{1}{8 \pi} \xi r^{\varrho} \sin \frac{\pi\left(\theta-\theta_{1}\right)}{\theta_{2}-\theta_{1}} \exp \left[-2 \gamma\left(2 s+\frac{1}{2} \sigma\right)\right] \\
& \times\left\{\left(\frac{x \exp (\sigma / 2)}{r}\right)^{\varrho+\gamma}-\left(\frac{x \exp (-\sigma / 2)}{r}\right)^{\varrho+\gamma}\right\} .
\end{aligned}
$$

From (3.17), (3.3) and the inequality $\varrho<\gamma$ we obtain (3.4) with $K_{1}$ given by (3.5).

4. Pólya peaks and the Phragmén-Lindelöf indicator. Combining Lemma 2.1, Lemma 3.1 and the notion of Pólya peaks, we shall obtain formal analogues of the Phragmén-Lindelöf indicator. This is of some independent interest; it also enables us to apply, to new situations, as important a tool as the subtrigonometric inequality.

We first introduce a real function $u(z)$ of the complex variable $z=r e^{i \theta}$.

I. The function $u(z)$ is defined and subharmonic in each sector

$$
\Delta_{m}: r_{m}^{\prime}<|z|<r_{m}^{\prime \prime}, \quad \alpha_{1}<\theta<\alpha_{2}, \quad\left(\alpha_{2}-\alpha_{1}<2 \pi\right) .
$$

The definition of $u(z)$ is extended to each boundary point $\zeta$ of $\Delta_{m}$ by

$$
u(\zeta)=\limsup _{z \rightarrow \zeta} u(z) \quad\left(z \in \Delta_{m}, \zeta \in \partial \Delta_{m}\right) .
$$

The sequences $\left\{r_{m}\right\},\left\{r_{m}^{\prime}\right\},\left\{r_{m}^{\prime \prime}\right\}$, and $\left\{\varepsilon_{m}\right\}$ satisfy the conditions (1.2) and (1.6). As a consequence, the sectors $\Delta_{m}$ are pairwise disjoint so that, although it is convenient to think of $u(z)$ as a single function, we may actually be dealing with a sequence of unrelated subharmonic functions.

II. Let $T(r)$ be a strictly positive function, nondecreasing and continuous on each interval $\left[r_{m}^{\prime}, r_{m}^{\prime \prime}\right]$. [ $T(r)$ need not be defined for $r \notin \Lambda$.]

Assume that

$$
\begin{aligned}
& T(r) \leqq\left(1+\varepsilon_{m}\right) T\left(r_{m}\right)\left(\frac{r}{r_{m}}\right)^{\varrho}=V_{m}\left(r_{m}\right)\left(\frac{r}{r_{m}}\right)^{\varrho}=V_{m}(r) \\
&\left(\varrho>0, r_{m}^{\prime} \leqq r \leqq r_{m}^{\prime \prime}, m=1,2,3, \ldots\right) .
\end{aligned}
$$


III. We now make full use of the notational conventions of $\S 1$ and consider $m=m(r)$ defined by (1.7). This clearly establishes the meaning of expressions such as

$$
\limsup _{r \rightarrow \infty} \frac{u\left(r e^{i \theta}\right)}{V(r)} \quad\left(r \in \Lambda, \alpha_{1}<\theta<\alpha_{2}\right) .
$$

The following Lemma 4.1 is a translation of Lemma 2.1 and Lemma 3.1 into the language of Pólya peaks.

L e m m 4.1. Let $u(z)$ and $T(r)$ be defined as above and let $V(r)=V_{m}(r)$ be the comparison function in (4.1).

Assume

$$
u(z) \leqq B V(r) \quad\left(|z|=r \in \Lambda, \alpha_{1}<\theta<\alpha_{2}\right),
$$

where $B \geqq 1$ is a constant.

Consider arguments $\theta_{1}, \theta_{2}$, such that

$$
\begin{gathered}
\alpha_{1} \leqq \theta_{1}<\theta_{2} \leqq \alpha_{2}, \quad \frac{\pi}{\gamma}=\theta_{2}-\theta_{1}<\frac{\pi}{\varrho}, \\
\limsup _{r \rightarrow \infty} \frac{u\left(r e^{\left.i \theta_{j}\right)}\right.}{V(r)} \leqq h_{j} \quad(r \in \Lambda, j=1,2),
\end{gathered}
$$

with $h_{1}$ and $h_{2}$ finite.

Let $s>0$ be given.

I. Then, if $H(\theta)$ is defined as in Lemma 2.1, we have

$$
u\left(r e^{i \theta}\right) \leqq\left\{\frac{r}{r_{m}}\right\}^{\varrho} T\left(r_{m}\right) H(\theta)+\eta_{m} T\left(r_{m}\right),
$$

uniformly for

$$
r \in \Lambda(s), \quad \theta_{1}<\theta<\theta_{2} .
$$

II. In addition to the preceding hypotheses, assume that there exist positive constants $\xi, \sigma$, and a positive sequence $\left\{x_{m}\right\}_{m}$, such that

$$
x_{m} \in I_{m}(s) \text {, }
$$

and such that for $j=1$, or for $j=2$,

$$
u\left(r e^{i \theta_{j}}\right) \leqq\left(h_{j}-\xi\right) V(r) \quad\left(x_{m} e^{-\sigma} \leqq r \leqq x_{m} e^{\sigma}\right) .
$$

Then,

$$
u\left(r e^{i \theta}\right) \leqq\left(\frac{r}{r_{m}}\right)^{e} T\left(r_{m}\right)\left\{H(\theta)-\xi K_{1} \sin \pi\left(\frac{\theta-\theta_{1}}{\theta_{2}-\theta_{1}}\right)+\eta_{m}\right\} \quad\left(r \in I_{m}(s)\right) .
$$


The value of $K_{1}$ is the same as in (3.5).

Proof. The lemma follows immediately from Lemmas 2.1 and 3.1 if the following points are borne in mind.

A. Take in Lemma 2.1

$$
l=r_{m}^{\prime}, \quad R=r_{m}^{\prime \prime}
$$

and notice that, in view of (4.1), (4.4) may be rewritten as $u\left(r e^{i \theta_{j}}\right) \leqq\left(h_{j}+\eta_{m}\right)\left(1+\varepsilon_{m}\right) \frac{T\left(r_{m}\right)}{r_{m}^{e}} r^{e} \quad\left(r_{m}^{\prime} \leqq r \leqq r_{m}^{\prime \prime}, \eta_{m}>0, \eta_{m} \rightarrow 0\right)$.

The implications of (4.2) are obvious. As to the term

$$
\left(\frac{l}{r}\right)^{e}+\left(\frac{r}{R}\right)^{\gamma-e}
$$

which appears in (2.7), it cannot exceed

$$
e^{s \varrho}\left(\frac{r_{m}^{\prime}}{r_{m}}\right)^{\varrho}+e^{s(\gamma-\varrho)}\left(\frac{r_{m}}{r_{m}^{\prime \prime}}\right)^{\gamma-\varrho}
$$

After some straightforward reductions, (1.2) and our convention about $\eta_{m}$ enable us to deduce (4.5) from (2.7).

B. The additional assumption (4.8) leads to

$$
u\left(r e^{i \theta_{j}}\right) \leqq\left(h_{j}+\eta_{m}-\xi\right)\left(1+\varepsilon_{m}\right) \frac{T\left(r_{m}\right)}{r_{m}^{o}} r^{o} \quad\left(x_{m} e^{-\sigma} \leqq r \leqq x_{m} e^{\sigma}\right),
$$

and Lemma 3.1 is clearly applicable. The positive parameters $s$ and $\sigma$ are the same as in Lemma 3.1, and from the conditions (4.7) we see that

$$
I_{m}(s) \subset\left[x_{m} e^{-2 s}, x_{m} e^{2 s}\right] .
$$

An inspection of (3.3) and (3.4) now shows that (4.9) is valid for $r \in I_{m}(s)$.

5. The Continuity Lemma. We sketch a proof for $f(z)$ nonconstant and meromorphic in the whole plane. Since we assume no normalization at the origin we require the additional condition

$$
R>R_{0}(f) .
$$

It is easily verified that

$$
\left|\log \alpha-\log ^{+} \beta\right| \leqq|\log \alpha-\log \beta| \quad(0 \leqq \alpha, 0 \leqq \beta, 0<\alpha+\beta) .
$$

If $\alpha+\beta=0$, the right-hand side of (5.2) is undefined; this is immaterial since the left-hand side is zero in this case. 
In order to obtain bounds for

$$
m(t, f ; J)-m\left(t^{\prime}, f ; J\right),
$$

as well as for

$$
m(t, f ; J)-m\left(t^{\prime}, f ; J\right),
$$

we write $\partial\left(t, t^{\prime}\right)$ for either one of the above differences. Hence, taking (5.2) into account, we deduce

$$
\left|\partial\left(t, t^{\prime}\right)\right| \leqq \frac{1}{2 \pi} \int_{-\pi}^{\pi}|\log | f\left(t e^{i \theta}\right)|-\log | f\left(t^{\prime} e^{i \theta}\right)|| d \theta .
$$

Write

$$
\begin{aligned}
& t e^{i \varphi}=z, \quad t^{\prime} e^{i \varphi}=z^{\prime}, \quad R / 2=s, \\
& \frac{s^{2}-t^{2}}{s^{2}+t^{2}-2 s t \cos \psi}=P(s, t, \psi) .
\end{aligned}
$$

By the Poisson - Jensen formula

$$
\begin{aligned}
& |\log | f(z)|-\log | f\left(z^{\prime}\right)|| \\
& \leqq \\
& \frac{1}{2 \pi} \int_{-\pi}^{\pi}|\log | f\left(s e^{i \theta}\right)||\left|P(s, t, \theta-\varphi)-P\left(s, t^{\prime}, \theta-\varphi\right)\right| d \theta \\
& \quad+\sum_{a}|\log | \frac{s^{2}-z^{\prime} \bar{a}}{s^{2}-z \bar{a}}||+\sum_{b}|\log | \frac{s^{2}-z \bar{b}}{s^{2}-z^{\prime} \bar{b}}|| \\
& \quad+\sum_{a}|\log | \frac{z-a}{z^{\prime}-a}||+\sum_{b}|\log | \frac{z-b}{z^{\prime}-b}||,
\end{aligned}
$$

where $a$ runs over the zeros of $f$ in $|z| \leqq s, b$ runs over the poles. It is easily seen that, under our restrictions on $t$ and $t^{\prime}$, there are absolute constants $A_{1}, A_{2}$ such that

$$
\begin{gathered}
\left|P(s, t, \psi)-P\left(s, t^{\prime}, \psi\right)\right| \leqq A_{1} \sigma \\
|\log | \frac{s^{2}-z^{\prime} \bar{d}}{s^{2}-z \bar{d}}|=| \log \left|1+\frac{\left(z-z^{\prime}\right) \bar{d}}{s^{2}-z \bar{d}}\right| \mid \leqq A_{2} \sigma \quad(|d| \leqq s),
\end{gathered}
$$

where $d$ denotes either $a$ or $b$.

Now

$$
n(s, f) \log 2 \leqq N(2 s, f) \leqq T(R, f) \quad\left(1 \leqq s=\frac{R}{2}\right),
$$

and by Nevanlinna's First Fundamental Theorem 


$$
m(s, f)+m\left(s, \frac{1}{f}\right) \leqq 2 T(R, f)+O(1)<3 T(R, f) \quad\left(R>R_{0}\right) .
$$

Combining the inequalities $(5.3)-(5.8)$, we verify the existence of an absolute constant $A_{3}>0$ such that

$$
\begin{aligned}
\left|\partial\left(t, t^{\prime}\right)\right| \leqq & A_{3} \sigma T(R, f)+\sum_{a} \frac{1}{2 \pi} \int_{-\pi}^{\pi}|\log | \frac{t e^{i \varphi}-a}{t^{\prime} e^{i \varphi}-a}|| d \varphi \\
& +\sum_{b} \frac{1}{2 \pi} \int_{-\pi}^{\pi}|\log | \frac{t^{\prime} e^{i \varphi}-b}{t e^{i \varphi}-b} \mid d \varphi .
\end{aligned}
$$

Clearly

$$
\left|\frac{z-d}{z^{\prime}-d}\right|=\left|1+\frac{z-z^{\prime}}{z^{\prime}-d}\right| \leqq 1+\frac{\left|t-t^{\prime}\right|}{\left|t^{\prime} e^{i \varphi}-d\right|} \quad\left(d=|d| e^{i \omega}\right),
$$

and since

$$
\left|z^{\prime}-d\right|=\left|t^{\prime} e^{i \varphi}-\right| d\left|e^{i \omega}\right| \geqq\left(t^{\prime}+|d|\right)\left|\sin \frac{\varphi-\omega}{2}\right|,
$$

we obtain

$$
\left|\frac{t e^{i \varphi}-d}{t^{\prime} e^{i \varphi}-d}\right| \leqq 1+\frac{\sigma}{\left|\sin \frac{\varphi-\omega}{2}\right|}
$$

This inequality holds if $t$ and $t^{\prime}$ are exchanged; consequently

$$
\begin{aligned}
& \int_{-\pi}^{\pi}|\log | \frac{t e^{i \varphi}-d}{t^{\prime} e^{i \varphi}-d}|| d \varphi \leqq 2 \int_{0}^{\pi} \log \left(1+\frac{\sigma}{\sin (\psi / 2)}\right) d \psi \\
& =4 \int_{0}^{\pi / 2} \log \left(1+\frac{\sigma}{\sin \varphi}\right) d \varphi<4 \int_{0}^{\pi / 2} \log \left(1+\frac{\pi \sigma}{2 \varphi}\right) d \varphi \\
& =2 \pi\left\{\log (1+\sigma)+\sigma \log \left(1+\frac{1}{\sigma}\right)\right\} \\
& <2 \pi\left((1+\log 2) \sigma+\sigma \log \frac{1}{\sigma}\right) .
\end{aligned}
$$

As in (5.7),

$$
\left\{n(s, f)+n\left(s, \frac{1}{f}\right)\right\} \log 2 \leqq 3 T(R, f) \quad\left(R>R_{0}\right) .
$$


Using (5.10) and (5.11) in (5.9) we deduce from the two interpretations of $\partial\left(t, t^{\prime}\right)$, both (31) and (32).

6. Equicontinuous character of $m^{*}(z)$ and $T^{*}(z)$. Using the Continuity Lemma of the present paper and a similar result which we established in [5; p. 337-339], Baernstein proved [1; pp. 422-424] that $m^{*}(z)$, defined by (21), is a continuous function of $z$. This does not quite suffice for our purpose and we prove the more precise

$\mathrm{L}$ e m m 6.1. Let $m^{*}(z)$ be associated with some nonconstant meromorphic function $f(z)$ and let $T(r)=T(r, f)$.

Let $t_{1}$ and $t_{2}$ satisfy all the following conditions

$$
\begin{aligned}
& 0<R_{0}<t_{j} \leqq \frac{R}{4} \quad(j=1,2), \\
& (1+\sigma)^{-1} \leqq \frac{t_{1}}{t_{2}} \leqq 1+\sigma \quad(0 \leqq \sigma) .
\end{aligned}
$$

Then

$$
\begin{aligned}
& \left|m^{*}\left(t_{1} e^{i \theta_{1}}\right)-m^{*}\left(t_{2} e^{i \theta_{2}}\right)\right| \\
& \leqq A T(R)\left\{\sigma\left(1+\log \frac{1}{\sigma}\right)+\left|\theta_{2}-\theta_{1}\right|\left(1+\log \frac{1}{\left|\theta_{2}-\theta_{1}\right|}\right)\right\} \\
& \quad\left(0 \leqq \theta_{1} \leqq \pi, 0 \leqq \theta_{2} \leqq \pi\right) .
\end{aligned}
$$

The relation (6.3) remains valid with its left-hand side replaced by

$$
\left|T *\left(t_{1} e^{i \theta_{1}}\right)-T *\left(t_{2} e^{i \theta_{2}}\right)\right|,
$$

and $A$ replaced by another absolute constant $A_{0}>0$.

Proof. Let $\varepsilon>0$ be given. By the definition (21) there exists some measurable set $E_{2}$ such that

$$
\left|E_{2}\right|=2 \theta_{2}, \quad m *\left(t_{2} e^{i \theta_{2}}\right)-\varepsilon \leqq m\left(t_{2}, f ; E_{2}\right) .
$$

We now determine $E_{1}$ so that

$$
\left|E_{1}\right|=2 \theta_{1}
$$

and

$$
E_{1} \subset E_{2} \text { if } \theta_{1} \leqq \theta_{2},
$$

or

$$
E_{2} \subset E_{1} \text { if } \theta_{2}<\theta_{1} \text {. }
$$

[The existence of such a set $E_{1}$ is readily verified.] By the Continuity Lemma, (6.1) and (6.2), 


$$
\left|m\left(t_{2}, f ; E_{2}\right)-m\left(t_{1}, f ; E_{2}\right)\right|<A T(R) \sigma\left(1+\log \frac{1}{\sigma}\right)
$$

If (6.5) holds take $E_{3}=E_{2}-E_{1}$; otherwise (6.6) holds and we take $E_{3}=E_{1}-E_{2}$. In either case

$$
\begin{aligned}
\left|m\left(t_{1}, f ; E_{2}\right)-m\left(t_{1}, f ; E_{1}\right)\right| & \leqq \frac{1}{2 \pi} \int_{E_{3}}|\log | f\left(t_{1} e^{i \theta}\right)|| d \theta \\
& \leqq m\left(t_{1}, f ; E_{3}\right)+m\left(t_{1}, \frac{1}{f} ; E_{3}\right) .
\end{aligned}
$$

We now use Lemma III of [5; p. 322], and take into account

$$
\left|E_{3}\right|=2\left|\theta_{2}-\theta_{1}\right|, \quad T\left(t, \frac{1}{f}\right) \leqq 2 T(t, f) \quad\left(t>R_{0}\right) .
$$

We thus find

$$
\left|m\left(t_{1}, f ; E_{2}\right)-m\left(t_{1}, f ; E_{1}\right)\right| \leqq A\left|\theta_{2}-\theta_{1}\right|\left(1+\stackrel{+}{+\infty} \frac{1}{\left|\theta_{2}-\theta_{1}\right|}\right) T(R) .
$$

Combining (6.4), (6.7), (6.8) and

$$
m\left(t_{1}, f ; E_{1}\right) \leqq m^{*}\left(t_{1} e^{i \theta_{1}}\right)
$$

we see that

$$
m *\left(t_{2} e^{i \theta 2}\right)-m^{*}\left(t_{1} e^{i \theta_{1}}\right)-\varepsilon
$$

is bounded from above by the right-hand side of (6.3). Since we may exchange in (6.9) $t_{1} e^{i \theta_{1}}$ and $t_{2} e^{i \theta_{2}}$ and since $\varepsilon(>0)$ is arbitrary, the inequality (6.3) follows.

The assertion concerning $T^{*}$, in Lemma 6.1 requires an upper bound for

$$
\left|N\left(t_{1}, f\right)-N\left(t_{2}, f\right)\right| \text {. }
$$

It is clearly no longer a restriction to assume $t_{1}>t_{2}>1$. Hence

$$
\begin{aligned}
0 & \leqq N\left(t_{1}, f\right)-N\left(t_{2}, f\right)=\int_{t_{2}}^{t_{1}} \frac{n(t, f)}{t} d t \leqq \frac{t_{1}-t_{2}}{t_{2}} n\left(t_{1}, f\right) \leqq \sigma n\left(t_{1}, f\right) \\
& \leqq \frac{\sigma}{\log 2} N\left(2 t_{1}, f\right) \leqq \frac{\sigma}{\log 2} T(R) .
\end{aligned}
$$

This is the required bound for $(6.10)$ and the last assertion of Lemma 6,1 is now obvious. 
Our next lemma is obtained by combining Lemma 6.1 and the peak inequality (1.3). It does not depend on any assumption concerning the extremal character of the spread.

$\mathrm{L}$ e $\mathrm{m} \mathrm{m}$ a 6.2. Let $T^{*}(z)$ be the Baernstein characteristic of some nonconstant meromorphic function $f(z)$. Put $T(r, f)=T(r)$ and let $\left\{r_{m}\right\}_{m}$ be a sequence of peaks of order $\varrho>0$ of $T(r)$.

Let

$$
T^{*}\left(t_{m} e^{i \omega_{m}}\right) \leqq \lambda_{m}\left(\frac{t_{m}}{r_{m}}\right)^{\varrho} T\left(r_{m}\right)
$$

where

$$
t_{m} \in I_{m}(s) \quad(s>0), \quad 0 \leqq \omega_{m} \leqq \pi, \quad m>m_{0},
$$

and

$$
\lambda_{m} \rightarrow \lambda \geqq 0, \quad \omega_{m} \rightarrow \omega \quad(m \rightarrow \infty) .
$$

Then, given $\xi>0$, it is possible to determine

$$
\sigma=\sigma(\lambda, \xi, \varrho, s)>0,
$$

depending on no parameters other than those explicitly listed, and such that

$$
T^{*}\left(t e^{i \theta}\right) \leqq(\lambda+\xi)\left(\frac{t}{r_{m}}\right)^{\varrho} T\left(r_{m}\right),
$$

provided

$$
e^{-\sigma} \leqq \frac{t}{t_{m}} \leqq e^{\sigma}
$$

and

$$
|\theta-\omega| \leqq \sigma, \quad 0 \leqq \theta \leqq \pi, \quad m>m_{0} .
$$

Proof. Take $\sigma$ to be the largest number satisfying simultaneously the three inequalities

$$
\begin{gathered}
0 \leqq \sigma \leqq 1 \\
\left(\lambda+\frac{\xi}{3}\right) e^{\sigma \varrho}+\frac{\xi}{3} \leqq \lambda+\xi \\
2 e 4^{\varrho+1} A_{0} \exp (2 \varrho(s+1)) \sigma\left(1+\log \frac{1}{\sigma}\right) \leqq \frac{\xi}{3} .
\end{gathered}
$$

In (6.20), $A_{0}$ is the absolute constant of Lemma 6.1 ; it is clear that $\sigma$ depends only on the parameters explicitly listed.

By (6.11), (6.13) and (6.16) 


$$
T^{*}\left(t_{m} e^{i \omega_{m}}\right) \leqq\left(\lambda+\frac{\xi}{3}\right) e^{\sigma \varrho}\left(\frac{t}{r_{m}}\right)^{\varrho} T\left(r_{m}\right) \quad\left(m>m_{0}\right) .
$$

By (6.12), (6.16) and (6.18),

$$
t \in I_{m}(s+\sigma) \subset I_{m}(s+1), \quad t_{m} \in I_{m}(s+1) .
$$

Hence, if we take

$$
R=4 e^{s+1} r_{m}
$$

the peak inequality (1.3) implies

$$
T(R) \leqq 4^{\varrho+1} \exp (\varrho(s+1)) T\left(r_{m}\right) \leqq 4^{\varrho+1} \exp (2 \varrho(s+1)) T\left(r_{m}\right)\left(\frac{t}{r_{m}}\right)^{\varrho} .
$$

By (6.13), (6.16), (6.17) and (6.18),

$$
\begin{aligned}
(1+e \sigma)^{-1} \leqq \frac{t}{t_{m}} \leqq(1+e \sigma), \quad\left|\theta-w_{m}\right| \leqq \sigma+\left|\omega-\omega_{m}\right| & \leqq e \sigma \\
& \left(m \geqq m_{0}\right) .
\end{aligned}
$$

Lemma 6.1, (6.20) and (6.22) immediately yield

$$
\begin{aligned}
& T^{*}\left(t e^{i \theta}\right)-T^{*}\left(t_{m} e^{i \omega_{m}}\right) \\
& \leqq A_{0} 4^{\varrho+1} \exp (2 \varrho(s+1)) T\left(r_{m}\right)\left(\frac{t}{r_{m}}\right)^{\varrho} 2 e \sigma\left(1+\log \frac{1}{\sigma}\right) \\
& \leqq \frac{\xi}{3}\left(\frac{t}{r_{m}}\right)^{\varrho} T\left(r_{m}\right) \quad\left(m>m_{0}\right) .
\end{aligned}
$$

We now combine (6.21) and (6.23) and take (6.19) into account. This leads to (6.15) and the lemma is proved.

7. An upper bound for $T^{*}\left(r e^{i \theta}\right)$. We apply Lemma 4.1, with $\varrho=\mu$ to $u(z)=T^{*}(z)$.

By the definition of $T^{*}$,

$$
\sup _{0 \leqq \theta \leqq \pi} T *\left(r e^{i \theta}\right)=T(r)=T(r, f)
$$

and

$$
T^{*}(r)=N(r, f) .
$$

Therefore, if $\delta(\infty, f)=1-\cos \beta \mu$, we find, in view of (4.1),

$$
\lim \sup \frac{T^{*}\left(r e^{i \beta}\right)}{V(r)} \leqq \lim \sup \frac{T^{*}\left(r e^{i \beta}\right)}{T(r)} \leqq 1 \quad(r \rightarrow \infty, r \in \Lambda),
$$




$$
\lim \sup \frac{T^{*}(r)}{V(r)} \leqq \lim \sup \frac{N(r, f)}{T(r)} \leqq \cos \beta \mu \quad(r \rightarrow \infty, r \in \Lambda)
$$

We now apply assertion I of Lemma 4.1 with

$$
\theta_{1}=0, \quad h_{1}=\cos \beta \mu ; \quad \theta<\theta_{2}=\beta^{*}=\min (\beta, \pi), \quad h_{2}=1,
$$

and find:

If $s>0$ is given and if

$$
r \in \Lambda(s), \quad 0<\theta<\min (\beta, \pi)
$$

then

$$
T^{*}\left(r e^{i \theta}\right) \leqq\left(\frac{r}{r_{m}}\right)^{\mu} T\left(r_{m}\right) \cos \mu(\beta-\theta)+\eta_{m} T\left(r_{m}\right) \quad(\beta<\pi)
$$

$$
\begin{array}{r}
\left(\frac{r}{r_{m}}\right)^{\mu} T\left(r_{m}\right)\left\{\cos \mu(\pi-\theta)-\frac{\cos \mu \pi-\cos \mu \beta}{\sin \mu \pi} \sin \mu(\pi-\theta)\right\}+\eta_{m} T\left(r_{m}\right) \\
(\pi \leqq \beta) .
\end{array}
$$

The relation (7.2) is essentially the same as the one used by Baernstein in his proof of the spread relation. The relation (7.3) is stated for sake of completeness. Its appearance is precluded by our hypotheses ES.

The following consequence of (7.2) will be needed later.

$\mathrm{R}$ e mark. It is possible to find three positive sequences $\left\{R_{m}^{\prime}\right\}$, $\left\{R_{m}^{\prime \prime}\right\},\left\{\tilde{\varepsilon}_{m}\right\}$, satisfying the conditions (18) and such that, for $\theta$ fixed $(0<\theta<\beta<\pi)$,

$$
\limsup _{r \rightarrow \infty} \frac{T^{*}\left(r e^{i \theta}\right)}{V(r)} \leqq \cos \mu(\beta-\theta) \quad\left(r \in \Lambda^{\prime}\right)
$$

with

$$
\Lambda^{\prime}=\bigcup_{m=1}^{\infty}\left[R_{m}^{\prime}, R_{m}^{\prime \prime}\right]
$$

The proof of the remark is immediate, we give to $s$, successively, the values $s=1,2,3, \ldots$ and complete the construction of $\left[R_{m}^{\prime}, R_{m}^{\prime \prime}\right]$ by an obvious "diagonalization".

8. The spread relation. We first prove that, if $\beta<\pi$, then

$$
\lim _{m \rightarrow \infty} \frac{T *\left(r_{m} e^{i \beta_{m}}\right)}{T\left(r_{m}\right)}=1
$$

with 


$$
\beta_{m}=\frac{1}{2}\left|E\left(r_{m}\right)\right|
$$

and

$$
E\left(r_{m}\right)=E=\left\{\theta: \log \left|f\left(r_{m} e^{i \theta}\right)\right| \geqq \eta\left(r_{m}\right) T\left(r_{m}\right)\right\} .
$$

Introduce, beside $E\left(r_{m}\right)$, the sets

$$
\begin{gathered}
E_{0}\left(r_{m}\right)=E_{0}=\left\{\theta: \log \left|f\left(r_{m} e^{i \theta}\right)\right| \geqq 0\right\}, \\
E^{+}=E_{0}-E \quad\left(\eta\left(r_{m}\right) \geqq 0\right), \\
E^{-}=E-E_{0} \quad\left(\eta\left(r_{m}\right)<0\right) .
\end{gathered}
$$

From (8.2), (8.3), (8.4) and (21) we deduce

$$
\begin{aligned}
m\left(r_{m}, f\right)-\left|\eta\left(r_{m}\right)\right| T\left(r_{m}\right) & \leqq \frac{1}{2 \pi} \int_{E} \log \left|f\left(r_{m} e^{i \theta}\right)\right| d \theta \\
& \leqq m^{*}\left(r_{m} e^{i \beta_{m}}\right) .
\end{aligned}
$$

Hence, taking into account (20) and (7.1), we find

$$
T\left(r_{m}\right)\left(1-\left|\eta\left(r_{m}\right)\right|\right) \leqq T^{*}\left(r_{m} e^{i \beta_{m}}\right) \leqq T\left(r_{m}\right),
$$

and (8.1) follows.

Up to this point we have not used the spread relation (5) or the hypotheses ES. It is therefore possible that

$$
\liminf _{m \rightarrow \infty} \beta_{m}<\beta \quad\left(0<\beta \leqq \frac{\pi}{2 \mu}, \beta<\pi\right),
$$

[the quantity $\beta$ is defined in (6)]. On the other hand, by (7.2) and (8.5), for suitable large values of $m$, we have

$$
T^{*}\left(r_{m} e^{i \beta}\right) \leqq T\left(r_{m}\right)\left(1+\eta_{m}\right) \cos \mu\left(\beta-\beta_{m}\right) .
$$

Since this contradicts (8.1), we must reject (8.5). This shows that our inequalities do in fact lead to a proof of the spread relation. The proof thus obtained does not differ essentially from Baernstein's original proof in [1].

9. Lower bound for $T^{*}\left(r e^{i \theta}\right)$. We now use (for the first time) the hypotheses ES. As remarked in the introduction these hypotheses imply

$$
0<\beta<\pi \text {. }
$$

In view of (12), (8.1) and Lemma 6.1 (with $\sigma=0$ ), they also yield

$$
\lim _{m \rightarrow \infty} \frac{T^{*}\left(r_{m} e^{i \beta}\right)}{T\left(r_{m}\right)}=1 \text {. }
$$


We now prove

L e $\mathrm{m} \mathrm{m}$ a 9.1. Let $f(z)$ satisfy the hypotheses ES. Then, given $s>0$, there exists a positive sequence $\left\{\eta_{m}\right\}_{m}$, independent of $r$ and $\theta$, and such that

$$
\eta_{m} \rightarrow 0 \quad(m \rightarrow \infty)
$$

and

(ii)

$$
T^{*}\left(r e^{i \theta}\right) \geqq\left(\frac{r}{r_{m}}\right)^{\mu} T\left(r_{m}\right)\left(\cos \mu(\beta-\theta)-\eta_{m}\right),
$$

provided

$$
r \in I_{m}(s), \quad 0 \leqq \theta \leqq \beta .
$$

Proof. Assume the lemma to be false. It is then possible to find $s>0$, $\xi>0$, infinitely many values of $m$ and associated points $x_{m} e^{i \omega_{m}}$ such that

$$
T^{*}\left(x_{m} e^{i \omega_{m}}\right) \leqq\left(\frac{x_{m}}{r_{m}}\right)^{\mu} T\left(r_{m}\right)\left(\cos \mu\left(\beta-\omega_{m}\right)-3 \xi\right),
$$

and

$$
x_{m} \in I_{m}(s), \quad 0 \leqq \omega_{m} \leqq \beta .
$$

Using, if necessary, the selection principle we may renumber the quantities $x_{m}, \omega_{m}$ and assume that

$$
\omega_{m} \rightarrow \omega \quad(m \rightarrow \infty), \quad 0 \leqq \omega \leqq \beta, \quad \cos \mu(\beta-\omega)-3 \xi \geqq 0 .
$$

Lemma 6.2 is immediately applicable and shows the existence of

$$
0<\sigma=\sigma(\mu, \beta, \omega, \xi, s) \leqq 1
$$

such that the relations

$$
e^{-\sigma} \leqq \frac{t}{x_{m}} \leqq e^{\sigma}, \quad|\theta-\omega| \leqq \sigma, \quad 0 \leqq \theta \leqq \beta, \quad m>m_{0},
$$

imply

$$
T^{*}\left(t e^{i \theta}\right) \leqq\left(\frac{t}{r_{m}}\right)^{\mu} T\left(r_{m}\right)(\cos \mu(\beta-\omega)-2 \xi) .
$$

In order to avoid the values $\omega=0$ and $\omega=\beta$, which complicate our arguments we take advantage of the fact that $\theta$ may vary in the interval $[\omega-\sigma, \omega+\sigma]$ and deduce from (9.8) the existence of some $\omega$ such that

$$
0<\omega<\beta,
$$

and 
(9.10)

$$
T^{*}\left(t e^{i \omega}\right) \leqq\left(\frac{t}{r_{m}}\right)^{\mu} T\left(r_{m}\right)(\cos \mu(\beta-\omega)-\xi) \leqq V(t)(\cos \mu(\beta-\omega)-\xi),
$$

for

$$
x_{m} e^{-\sigma} \leqq t \leqq x_{m} e^{\sigma}, \quad m>m_{0} .
$$

We now apply Lemma 4.1, II with

$$
\theta_{1}=\omega, \quad h_{1}=\cos \mu(\beta-\omega) ; \quad \theta=\beta ; \quad \beta<\theta_{2}<\frac{3 \beta}{2}, \quad h_{2}=1 ;
$$

as to the parameters $\xi, s$ and $\sigma$ we remark that: $\xi$ and $s$ are determined by (9.4), and $\sigma(0<\sigma \leqq 1)$, whose value is determined by Lemma 6.2, only depends on the parameters explicitly listed in (9.6).

Write

$$
\frac{\pi}{\gamma}=\psi=\theta_{2}-\theta_{1}=\theta_{2}-\omega
$$

and notice that, by (9.9) and (9.11)

$$
\frac{2 \pi}{3 \beta}<\gamma<\frac{\pi}{\beta-\omega} .
$$

By (7.1) the choice $h_{2}=1$ is certainly possible, regardless of the final selection of $\theta_{2}$. The possibility of selecting $h_{1}$, as in (9.11) is derived from a preliminary application of (7.2) with $\theta=\omega$. In order to completely justify this step, we observe that, as stated, (7.2) only yields

$$
\limsup _{r \rightarrow \infty} \frac{T^{*}\left(r e^{i \theta}\right)}{V(r)} \leqq \cos \mu(\beta-\theta) \quad(r \in \Lambda(s)),
$$

where $s>0$ is fixed. On the other hand, condition (4.4), which is needed to permit the use of Lemma 4.1, requires that $\Lambda(s)$ be replaced by $\Lambda$ in (9.13). The remark, at the end of $\S 7$, shows that the validity of Lemma 4.1 is not affected if $\Lambda$ is replaced by $\Lambda^{\prime}$. Hence, in view of $(9.10)$ and (9.11), we deduce from (4.9)

$$
T^{*}\left(r_{m} e^{i \beta}\right) \leqq T\left(r_{m}\right)\left\{H(\beta)-\xi K_{1} \sin \pi\left(\frac{\beta-\omega}{\psi}\right)+\eta_{m}\right\},
$$

where

$$
H(\beta)=\frac{\cos \mu(\beta-\omega) \sin \mu(\psi+\omega-\beta)+\sin \mu(\beta-\omega)}{\sin \mu \psi}
$$


and $K_{1}$ is given by (3.5). In order to replace $K_{1}$ by a constant independent of $\gamma$ (and therefore independent of $\psi$ ), we notice that (3.5) and (9.12) imply

$$
\xi K_{1} \geqq \xi(8 \pi)^{-1} \exp \left(-4 \frac{\pi}{\beta-\omega}(2 s+\sigma)\right)\left\{\exp \left(\frac{2 \pi \sigma}{3 \beta}\right)-1\right\}=K_{2} .
$$

This suggests the introduction of the function of $\psi$ :

$$
\mathscr{L}(\psi)=H(\beta)-K_{2} \sin \pi\left(\frac{\beta-\omega}{\psi}\right) \quad(\beta \text { fixed }) .
$$

From (9.14), (9.16) and (9.17) we deduce

$$
T^{*}\left(r_{m} e^{i \beta}\right) \leqq T\left(r_{m}\right)\left\{\mathscr{L}(\psi)+\eta_{m}\right\} .
$$

Considered as a real analytic function of $\psi, \mathscr{L}(\psi)$ is regular for

$$
|\psi-\beta+\omega| \leqq \frac{\beta-\omega}{2},
$$

and hence there exists a bound $K_{3}$, independent of $\psi$ and such that

$$
\left|\mathscr{L}^{\prime \prime}(\psi)\right| \leqq 2 K_{3},
$$

for $\psi$ restricted by (9.19). Since

$$
\mathscr{L}(\beta-\omega)=1, \quad \mathscr{L}^{\prime}(\beta-\omega)=-K_{2} \frac{\pi}{\beta-\omega},
$$

the restrictions (9.19) also imply

$$
\mathscr{L}(\psi) \leqq 1-K_{2} \frac{\pi}{\beta-\omega}(\psi-\beta+\omega)+K_{3}(\psi-\beta+\omega)^{2} .
$$

It is now clear that, if $\psi-\beta+\omega$ is chosen positive and small enough, we have

$$
\mathscr{L}(\psi)<1
$$

and by $(9.18)$

$$
\limsup _{m \rightarrow \infty} \frac{T^{*}\left(r_{m} e^{i \beta}\right)}{T\left(r_{m}\right)}<1 .
$$

Since this contradicts (9.2) we must reject (9.4) and Lemma 9.1 is proved.

10. Proof of Theorems 1 and 2. Using Lemma 9.1 with $\theta=\beta$, and the peak inequality, we obtain 


$$
\begin{aligned}
\left(1-\eta_{m}\right)\left(\frac{r}{r_{m}}\right)^{\mu} T\left(r_{m}\right) & \leqq T^{*}\left(r e^{i \beta}\right) \leqq T(r) \\
& \leqq\left(1+\varepsilon_{m}\right)\left(\frac{r}{r_{m}}\right)^{\mu} T\left(r_{m}\right) \quad(r \in \Lambda(s)) .
\end{aligned}
$$

This implies (15).

To prove (16) use Lemma 9.1 with $\theta=0$. Then

$$
\left(\cos \mu \beta-\eta_{m}\right)\left(\frac{r}{r_{m}}\right)^{\mu} T\left(r_{m}\right) \leqq N(r, f) \quad\left(r \in I_{m}(s)\right),
$$

and, in view of (15), we find

$$
\cos \mu \beta \leqq \liminf _{\substack{r \rightarrow \infty \\ r \in \Lambda(s)}} \frac{N(r, f)}{T(r)} \leqq \limsup _{r \rightarrow \infty} \frac{N(r, f)}{T(r)}=\cos \mu \beta .
$$

This yields (16).

As to (17), it readily follows from (15), (16), the elementary inequalities

$$
\frac{\sigma-1}{\sigma} \frac{n(t, f)}{T(t)} \leqq \frac{N(\sigma t, f)-N(t, f)}{T(t)} \leqq(\sigma-1) \frac{n(\sigma t, f)}{T(t)} \quad(\sigma>1),
$$

and two successive passages to the limit: first $t \rightarrow \infty \quad(t \in \Lambda(s))$, then $\sigma \rightarrow 1+$.

The proof of Theorem 1 is now complete. To obtain Theorem 2 it suffices to combine (15), (7.2) and Lemma 9.1.

\section{A fundamental identity and some of its consequences.} Let $f(z)$ be a meromorphic function with zeros $\left\{a_{j}\right\}_{j}$ and poles $\left\{b_{j}\right\}_{j}$. As usual, multiplicities are taken into account by a suitable repetition of elements in these sequences. We first state as Lemma A an identity which has been proved by one of us $[6 ; \mathrm{p} .18]$. An essentially equivalent result was used by V.P. Petrenko [9].

L e $\mathrm{m} \mathrm{m}$ a A. Let $f(z)$ be meromorphic in

$$
D: 0<|z| \leqq R, \quad|\arg z| \leqq \frac{\pi}{\gamma} \quad(\gamma>1) .
$$

Let $0<u<R$ and put

$$
\begin{aligned}
& S_{1}=S_{1}(R, \gamma)=\frac{\gamma^{2}}{2 \pi} \int_{0}^{R} d t \frac{u^{\gamma} t^{\gamma-1}\left(R^{2 \gamma}-t^{2 \gamma}\right)\left(R^{2 \gamma}-u^{2 \gamma}\right)}{\left(t^{\gamma}+u^{\gamma}\right)^{2}\left(R^{2 \gamma}+u^{\gamma} t^{\gamma}\right)^{2}} \int_{-\pi / \gamma}^{\pi / \gamma} \log \left|f\left(t e^{i \theta}\right)\right| d \theta, \\
& (11.2) \\
& S_{2}=S_{2}(R, \gamma)=\frac{\gamma}{\pi} \int_{-\pi / \gamma}^{\pi / \gamma} \log \left|f\left(R e^{i \theta}\right)\right| \frac{R^{\gamma} u^{\gamma}\left(R^{\gamma}-u^{\gamma}\right)(1+\cos \gamma \theta) d \theta}{\left(R^{\gamma}+u^{\gamma}\right)\left(R^{2 \gamma}+u^{2 \gamma}-2 R^{\gamma} u^{\gamma} \cos \gamma \theta\right)},
\end{aligned}
$$




$$
W(z)=\log \frac{|z|^{\gamma}+u^{\gamma}}{\left|z^{\gamma}-u^{\gamma}\right|}-\log \frac{R^{2 \gamma}+|z|^{\gamma} u^{\gamma}}{\left|R^{2 \gamma}-z^{\gamma} u^{\gamma}\right|} .
$$

Then, if $f(u) \neq 0, f(u) \neq \infty$, we have

$$
\log |f(u)|=S_{1}+S_{2}+\sum_{b} W(b)-\sum_{a} W(a),
$$

where $\sum_{b}$ is summation over all poles $b$ of $f(z)$ in $D, \sum_{a}$ is summation over all zeros a of $f(z)$ in $D$.

The quantities $W(a)$ and $W(b)$ are nonnegative.

The formula still holds for $\gamma=1$ and does not require that $f\left(e^{i \pi} r\right)$ and $f\left(e^{-i \pi} r\right)$ be equal.

From Lemma A we deduce

L e m m a 11.1. Let $f(z)$ be a nonrational meromorphic function, normalized by the condition $f(0)=1$.

Assume $\gamma \geqq 1$ and let

$$
\begin{gathered}
J=\left\{\varphi:|\varphi| \leqq \frac{\pi}{\gamma}\right\}, \\
X(t, u)=\frac{t^{\gamma} u^{\gamma}}{\left(t^{\gamma}+u^{\gamma}\right)^{2}}, \\
H(z, \zeta)=\log \frac{|z|^{\gamma}+|\zeta|^{\gamma}}{\left|z^{\gamma}-\zeta^{\gamma}\right|},
\end{gathered}
$$

where $\zeta$ may be complex.

Then, if $f(u) \neq 0, f(u) \neq \infty$, we have

$$
\begin{aligned}
& \log |f(u)|+\gamma^{2} \int_{R^{\prime}}^{R} m\left(t, \frac{1}{f} ; J\right) X(t, u) \frac{d t}{t}+\sum_{\substack{a_{j} \in D \\
R^{\prime}<\left|a_{j}\right|}} H\left(a_{j}, u\right) \\
& =\gamma^{2} \int_{R^{\prime}}^{R} m(t, f ; J) X(t, u) \frac{d t}{t}+\sum_{\substack{b_{j} \in D \\
R^{\prime}<\left|b_{j}\right|}} H\left(b_{j}, u\right)+S,
\end{aligned}
$$

where

$$
|S| \leqq 28 \gamma\left\{\left(\frac{R^{\prime}}{u}\right)^{\gamma} T\left(2 R^{\prime}\right)+\left(\frac{u}{R}\right)^{\gamma} T(2 R)\right\}
$$

provided

$$
0<2 R^{\prime} \leqq u \leqq \frac{R}{2}
$$

Proof. Since $f(0)=1$, Jensen's formula is 
(11.11)

$$
m(r, f)+N(r, f)=m\left(r, \frac{1}{f}\right)+N\left(r, \frac{1}{f}\right)=T(r) \quad(r>0),
$$

and hence, from (11.2) and (11.10) we deduce

$$
\left|S_{2}\right| \leqq \frac{4 \gamma R^{\gamma} u^{\gamma}}{R^{2 \gamma}-u^{2 \gamma}}\left\{m(R, f)+m\left(R, \frac{1}{f}\right)\right\} \leqq 16 \gamma\left(\frac{u}{R}\right)^{\gamma} T(R) .
$$

Put

$$
Y(t, u ; R)=\frac{R^{2 \gamma} t^{\gamma} u^{\gamma}}{\left(R^{2 \gamma}+t^{\gamma} u^{\gamma}\right)^{2}},
$$

and rewrite $S_{1}$ in the form

$$
S_{1}=\gamma^{2} \int_{0}^{R}\left\{m(t, f ; J)-m\left(t, \frac{1}{f} ; J\right)\right\}[X(t, u)-Y(t, u ; R)] \frac{d t}{t},
$$

$$
S_{1}=\gamma^{2} \int_{0}^{R}\left\{m(t, f ; J)-m\left(t, \frac{1}{f} ; J\right)\right\} X(t, u) \frac{d t}{t}+S_{1}^{*}
$$

By (11.11) and (11.13)

$$
\left|S_{1}^{*}\right| \leqq 2 \gamma\left(\frac{u}{R}\right)^{\gamma} T(R) .
$$

Similarly

$$
\left|\gamma^{2} \int_{0}^{R^{\prime}}\left\{m(t, f ; J)-m\left(t, \frac{1}{f} ; J\right)\right\} X(t, u) \frac{d t}{t}\right| \leqq 2 \gamma\left(\frac{R^{\prime}}{u}\right)^{\gamma} T\left(R^{\prime}\right) .
$$

From (11.14), (11.15) and (11.16) we deduce

$$
\begin{gathered}
\left|S_{1}-\gamma^{2} \int_{R^{\prime}}^{R}\left\{m(t, f ; J)-m\left(t, \frac{1}{f} ; J\right)\right\} X(t, u) \frac{d t}{t}\right| \\
\leqq 2 \gamma\left\{\left(\frac{R^{\prime}}{u}\right)^{\gamma} T\left(R^{\prime}\right)+\left(\frac{u}{R}\right)^{\gamma} T(R)\right\} .
\end{gathered}
$$

By (11.3) and (11.7)

$$
0 \leqq \sum_{a_{j} \in D} W\left(a_{j}\right) \leqq \sum_{\substack{a_{j} \in D \\\left|a_{j}\right| \leqq R^{\prime}}} H\left(a_{j}, u\right)+\sum_{\substack{a_{j} \in D \\ R^{\prime}<\left|a_{j}\right|}}^{\prime} H\left(a_{j}, u\right)
$$


and obviously

$$
0 \leqq H(z, u)=\log \frac{|z|^{\gamma}+u^{\gamma}}{\left|z^{\gamma}-u^{\gamma}\right|} \leqq \frac{8}{3}\left|\frac{z}{u}\right|^{\gamma} \quad\left(|z| \leqq R^{\prime} \leqq \frac{u}{2}\right) .
$$

Similarly

$$
0 \leqq \log \frac{R^{2 \gamma}+|z|^{\gamma} u^{\gamma}}{\left|R^{2 \gamma}-z^{\gamma} u^{\gamma}\right|} \leqq \frac{8}{3}\left(\frac{u}{R}\right)^{\gamma} \quad\left(u \leqq \frac{R}{2},|z| \leqq R\right),
$$

and hence, returning to (11.3), we find, in view of (11.18) and (11.19)

$$
\begin{aligned}
-\frac{8}{3}\left(\frac{u}{R}\right)^{\gamma} n\left(R, \frac{1}{f}\right) & \leqq \sum_{a_{j} \in D} W\left(a_{j}\right)-\sum_{\substack{a_{j} \in D \\
R^{\prime}<\left|a_{j}\right|}} H\left(a_{j}, u\right) \\
& \leqq \frac{8}{3}\left|\frac{R^{\prime}}{u}\right|^{\gamma} n\left(R^{\prime}, \frac{1}{f}\right) .
\end{aligned}
$$

The familiar consequence of (11.11):

$$
n\left(r, \frac{1}{f}\right) \log 2 \leqq \int_{r}^{2 r} \frac{n\left(t, \frac{1}{f}\right)}{t} d t \leqq N\left(2 r, \frac{1}{f}\right) \leqq T(2 r) \quad(r>0),
$$

is used to eliminate from (11.20) the terms involving the function $n(r, 1 / f)$; we thus obtain

$$
\left|\sum_{a_{j} \in D} W\left(a_{j}\right)-\sum_{\substack{a_{j} \in D \\ R^{\prime}<\left|a_{j}\right|}} H\left(a_{j}, u\right)\right| \leqq 5\left\{\left(\frac{u}{R}\right)^{\gamma} T(2 R)+\left(\frac{R^{\prime}}{u}\right)^{\gamma} T\left(2 R^{\prime}\right)\right\} .
$$

There is a similar relation with the zeros $a$ replaced by the poles $b$.

The estimate (11.9) follows from (11.4), (11.12), (11.17) and (11.22). This completes the proof of Lemma 11.1.

L e $\mathrm{m} \mathrm{m}$ a 11.2. Let $f(z)$ be meromorphic and nonrational.

Let $\left\{R_{m}^{\prime}\right\},\left\{R_{m}^{\prime \prime}\right\}$ be sequences such that

$$
R_{m}^{\prime} \rightarrow \infty \quad(m \rightarrow \infty), \quad 4 R_{m}^{\prime}<R_{m}^{\prime \prime},
$$

and let $\left\{\omega_{m}\right\}_{m}$ be some given sequence of arguments.

Introduce the sectors

$$
\mathscr{D}_{m}=\left\{z: R_{m}^{\prime}<|z| \leqq R_{m}^{\prime \prime}, \omega_{m}-\frac{\pi}{\gamma} \leqq \arg z \leqq \omega_{m}+\frac{\pi}{\gamma}\right\}
$$

and the notation 


$$
\frac{1}{2 \pi} \int_{\omega_{m}-\pi / \gamma}^{\omega_{m}+\pi / \gamma} \log \left|f\left(r e^{i \theta}\right)\right| d \theta=m\left(r, f ;\left[\omega_{m}-\frac{\pi}{\gamma}, \omega_{m}+\frac{\pi}{\gamma}\right]\right),
$$

where

$$
\gamma \geq 1
$$

If

$$
2 R_{m}^{\prime} \leqq u \leqq \frac{1}{2} R_{m}^{\prime \prime}
$$

then

$$
\begin{aligned}
& \log \left|f\left(u e^{i \omega_{m}}\right)\right|+\sum_{a_{j} \in \mathscr{D}_{m}} H\left(a_{j} e^{-i \omega_{m}}, u\right) \\
& =\gamma^{2} \int_{R_{m}^{\prime}}^{R_{m}^{\prime \prime}} m\left(t, f ;\left[\omega_{m}-\frac{\pi}{\gamma}, \omega_{m}+\frac{\pi}{\gamma}\right]\right) X(t, u) \frac{d t}{t} \\
& +\sum_{b_{j} \in \mathscr{D}_{m}} H\left(b_{j} e^{-i \omega_{m}}, u\right)+S_{m}^{*} \quad\left(m>m_{0}\right),
\end{aligned}
$$

with

$$
\left|S_{m}^{*}\right| \leqq 56 \gamma\left\{\left(\frac{R_{m}^{\prime}}{u}\right)^{\gamma} T\left(2 R_{m}^{\prime}\right)+\left(\frac{u}{R_{m}^{\prime \prime}}\right)^{\gamma} T\left(2 R_{m}^{\prime \prime}\right)\right\}+K \log u .
$$

The constant $K>0$ only depends on the behavior of $f(z)$ at the origin.

Proof. Since the normalization $f(0)=1$ has been omitted, we first define an auxiliary function $F(z)$ by the conditions

$$
f(z)=c z^{k} F(z)
$$

where $k$ is an integer and

$$
c \neq 0, \quad F(0)=1 .
$$

Put

$$
T_{1}(r)=T(r, F), \quad T(r)=T(r, f) .
$$

Apply Lemma 11.1 to the auxiliary function

$$
F_{m}(z)=F\left(z e^{i \omega_{m}}\right)
$$

with

$$
R^{\prime}=R_{m}^{\prime}, \quad R=R_{m}^{\prime \prime} .
$$

All the necessary conditions are clearly satisfied and hence, in view of (11.26) and (11.27), we deduce from (11.8) 
(11.28) $\log \left|f\left(u e^{i \omega_{m}}\right)\right|-\log |c|-k \log u+\sum_{a_{j} \in \mathscr{D}_{m}} H\left(a_{j} e^{-i \omega_{m}}, u\right)$

$$
\begin{aligned}
& =\gamma^{2} \int_{R_{m}^{\prime}}^{R_{m}^{\prime \prime}} m\left(t, f ;\left[\omega_{m}-\frac{\pi}{\gamma}, \omega_{m}+\frac{\pi}{\gamma}\right]\right) X(t, u) \frac{d t}{t} \\
& -\gamma \int_{R_{m}^{\prime}}^{R_{m}^{\prime \prime}}(\log |c|+k \log t) X(t, u) \frac{d t}{t}+\sum_{b_{j} \in \mathscr{D}_{m}} H\left(b_{j} e^{-i \omega_{m}}, u\right)+S_{m}
\end{aligned}
$$

with

$$
\left|S_{m}\right| \leqq 28 \gamma\left\{\left(\frac{R_{m}^{\prime}}{u}\right)^{\gamma} T_{1}\left(2 R_{m}^{\prime}\right)+\left(\frac{u}{R_{m}^{\prime \prime}}\right)^{\gamma} T_{1}\left(2 R_{m}^{\prime \prime}\right)\right\} .
$$

To justify (11.29) it is necessary to observe that

$$
T_{1}(r)=T(r, F(z))=T\left(r, F\left(e^{i \omega} z\right)\right)
$$

for any choice of $\omega$ (real). We also note that, since $f(z)$ is not rational,

$$
T_{1}(r) \leqq 2 T(r) \quad\left(r>r_{0}\right)
$$

The assumption $\gamma \geqq 1$ implies the convergence of the integrals in the following elementary relation

$$
\left|\int_{R_{m}^{\prime}}^{R_{m}^{\prime \prime}} X(t, u) \frac{\log t}{t} d t\right| \leqq \log u \int_{0}^{\infty} \frac{r^{\gamma-1}}{\left(1+r^{\gamma}\right)^{2}} d r+\int_{0}^{\infty}|\log r| \frac{r^{\gamma-1}}{\left(1+r^{\gamma}\right)^{2}} d r .
$$

Now use (11.30), (11.31) and $R_{m}^{\prime} \rightarrow+\infty$ in (11.28) and (11.29). This leads to (11.24) and (11.25) and completes the proof of Lemma 11.2.

12. Estimates for $H(z, \zeta)$. We study

$$
H(z, \zeta)=\log \left|\frac{|z|^{\gamma}+|\zeta|^{\gamma}}{z^{\gamma}-\zeta^{\gamma}}\right|
$$

with

$$
\gamma \geqq 1, \quad \zeta=u e^{i \psi} \quad(u \geqq 0), \quad z=r e^{i \theta},
$$

and first state the obvious relations

$$
0 \leqq H(z, \zeta)=H(\zeta, z) \leqq H(|z|,|\zeta|) .
$$

$\mathrm{L}$ e $\mathrm{m} \mathrm{m}$ a 12.1. Let $\psi=0, s>0$, 


$$
e^{-s} u \leqq r \leqq e^{s} u
$$

and

$$
|\theta| \leqq \frac{\pi-\delta}{\gamma} \quad(0<\delta \leqq \pi)
$$

Then

$$
H(z, u) \geqq e^{s \gamma}\left(1+e^{s \gamma}\right)^{-2} \sin ^{2} \frac{\delta}{2}
$$

If, instead of (12.5), we assume

$$
0<\frac{\delta}{\gamma} \leqq|\theta| \leqq \frac{2 \pi-\delta}{\gamma},
$$

then

$$
H(|z|, u)-H(z, u) \geqq e^{s \gamma}\left(1+e^{s \gamma}\right)^{-2} \sin ^{2} \frac{\delta}{2} .
$$

Proof. From (12.1) and (12.5) we deduce

$$
H(z, u)=\frac{1}{2} \log \left(1+\frac{4 r^{\gamma} u^{\gamma} \cos ^{2}(\gamma \theta / 2)}{\left|z^{\gamma}-u^{\gamma}\right|^{2}}\right) \geqq \frac{r^{\gamma} u^{\gamma} \sin ^{2}(\delta / 2)}{\left(r^{\gamma}+u^{\gamma}\right)^{2}},
$$

and, using (12.4), we obtain (12.6).

Similarly, using (12.7), we find

$$
H(|z|, u)-H(z, u)=\frac{1}{2} \log \left(1+\frac{4 r^{\gamma} u^{\gamma} \sin ^{2}(\gamma \theta / 2)}{\left(r^{\gamma}-u^{\gamma}\right)^{2}}\right) \geqq \frac{r^{\gamma} u^{\gamma} \sin ^{2}(\delta / 2)}{\left(r^{\gamma}+u^{\gamma}\right)^{2}}
$$

and (12.8) follows.

In our next estimate we require Cartan's lemma on the minimum modulus of a polynomial.

$\mathrm{L}$ e $\mathrm{m} \mathrm{m}$ a 12.2. Let $\sigma(0<\sigma<1)$ be given and let $t_{j}(j=1,2, \ldots, \mathscr{N})$ be real numbers, not necessarily distinct, such that

$$
0 \leqq t_{j} \leqq r e^{2 \sigma} \quad(0<j \leqq \mathscr{N}) .
$$

Then, for some $u$ in the interval

$$
r \leqq u \leqq e^{\sigma} r,
$$

we have

$$
\Sigma_{1}=\sum_{j=1}^{\mathscr{N}} H\left(t_{j}, u\right)=\sum_{j=1}^{\mathscr{N}} \log \left(\frac{t_{j}^{\gamma}+u^{\gamma}}{\left|t_{j}^{\gamma}-u^{\gamma}\right|}\right) \leqq \mathscr{N}\left(K_{4}(\gamma)+\log \frac{1}{\sigma}\right)
$$

with 


$$
K_{4}(\gamma)=\log \left(\frac{8}{\gamma \delta} e^{2 \gamma+1}\right)
$$

Proof. Put

$$
x=u^{\gamma} .
$$

By (12.10)

$$
r^{\gamma} \leqq x \leqq r^{\gamma} e^{\sigma \gamma}
$$

From (12.9) and (12.10)

$$
\Sigma_{1} \leqq \mathscr{N} \log \left(2 r^{\gamma} \exp (2 \sigma \gamma)\right)+\log \frac{1}{|P(x)|},
$$

with

$$
P(x)=\prod_{j=1}^{\mathscr{N}}\left(x-t_{j}^{\gamma}\right)
$$

By Cartan's lemma, it is possible to exclude, from the interval of $x$ defined by (12.12), an exceptional set $\mathscr{E}$ such that

$$
|\mathscr{E}| \leqq \gamma \sigma r^{\gamma}
$$

and such that, for $x \notin \mathscr{E}$,

$$
\log \frac{1}{|P(x)|} \leqq \mathscr{N} \log \left(r^{-\gamma}\right)+\mathscr{N} \log \left(\frac{4 e}{\gamma \sigma}\right) .
$$

Combining (12.13) and (12.15), we obtain (12.11) for certain values of $u$ in the interval (12.10). The set of suitable values of $u$ has positive measure since, by (12.14),

$$
|\mathscr{E}|<r^{\gamma}\left(e^{\gamma \sigma}-1\right) .
$$

13. Further consequences of the hypotheses ES. If $f(z)$ satisfies the hypotheses ES, Theorem 1 is valid. Hence (17), restated in the same form as (19), enables us to determine sequences $\left\{R_{m}^{\prime}\right\},\left\{R_{m}^{\prime \prime}\right\}$ such that

$$
n(t, f)=\left(\frac{t}{r_{m}}\right)^{\mu}(B+\eta(t)) T\left(r_{m}\right) \quad(B=\mu \cos \beta \mu),
$$

with

$$
R_{m}^{\prime} \rightarrow \infty, \quad \frac{r_{m}}{R_{m}^{\prime}} \rightarrow \infty, \quad \frac{R_{m}^{\prime \prime}}{r_{m}} \rightarrow \infty \quad(m \rightarrow \infty)
$$

and 


$$
\eta(t) \rightarrow 0
$$

as $t \rightarrow \infty$ by values

$$
t \in \Lambda^{\prime}=\bigcup_{m=1}^{\infty}\left[R_{m}^{\prime}, R_{m}^{\prime \prime}\right] \quad\left(r_{m}^{\prime}<R_{m}^{\prime}, R_{m}^{\prime \prime}<r_{m}^{\prime \prime}\right)
$$

From this point on, the parameter $\gamma$ which appears in the definition of $H(z, \zeta)$ is always taken to be

$$
\gamma=\frac{\pi}{\beta}
$$

In view of the hypotheses $\mathrm{ES}$, it is obvious that

$$
\gamma>1, \quad \gamma \geqq 2 \mu>0 \text {. }
$$

L e $\mathrm{mm}$ a 13.1. Let $f(z)$ be a meromorphic function satisfying the hypotheses $E S$, at the sequence of peaks $\left\{r_{m}\right\}_{m}$, of order $\mu$, of $T(r)=T(r, f)$.

Assume that the quantities $R_{m}^{\prime}, R_{m}^{\prime \prime}$ have been selected as above.

Let $s>0$ and $\sigma \quad(0<\sigma<1)$ be given; let $x$ and $u$ be any two points such that

$$
x \in I_{m}(s), \quad x \leqq u \leqq e^{\sigma} x
$$

and let

$$
\begin{aligned}
& \Sigma_{0}(u)=\sum_{R_{m}^{\prime}<\left|b_{j}\right| \leqq x e^{-\sigma}} H\left(\left|b_{j}\right|, u\right), \\
& \Sigma_{2}(u)=\sum_{x e^{2 \sigma}<\left|b_{j}\right| \leqq R_{m}^{\prime \prime}} H\left(\left|b_{j}\right|, u\right) .
\end{aligned}
$$

Then, as $u \rightarrow \infty$

$\Sigma_{0}(u)+\Sigma_{2}(u) \leqq\left(\frac{u}{r_{m}}\right)^{\mu} T\left(r_{m}\right)\left(\pi B \tan \left(\frac{\pi \mu}{2 \gamma}\right)+\eta_{m}\right) \quad\left(\eta_{m} \rightarrow 0, m=m(u)\right)$.

Proof. From (13.1) we deduce

$$
n(t)-B \frac{T\left(r_{m}\right)}{r_{m}^{\mu}} t^{\mu}=\eta(t) \frac{T\left(r_{m}\right)}{r_{m}^{\mu}} t^{\mu}=\xi(t) \quad(n(t)=n(t, f)),
$$

with

$$
\eta(t) \rightarrow 0, \quad\left(t \rightarrow \infty, t \in \Lambda^{\prime}\right)
$$

The function $\xi(t)$ is expressed, in (13.9), as the difference of two nondecreasing functions; hence the symbol $d \xi$ has a welldefined meaning. Put 


$$
\begin{aligned}
& \eta_{m}=\sup _{R_{m}^{\prime} \leqq t \leqq R_{m}^{\prime \prime}}|\eta(t)|, \\
& \tau=x e^{2 \sigma}, \quad R^{\prime \prime}=R_{m}^{\prime \prime} .
\end{aligned}
$$

Using Stieltjes integrals and (13.9), we obtain

$$
\Sigma_{2}(u)=\int_{\tau+}^{R^{\prime \prime}} H(t, u) d n(t)=\mu B \frac{T\left(r_{m}\right)}{r_{m}^{\mu}} \int_{\tau}^{R^{\prime \prime}} H(t, u) t^{\mu-1} d t+\int_{\tau+}^{R^{\prime \prime}} H(t, u) d \xi .
$$

An integration by parts and (13.10) yield

$$
\begin{aligned}
& \left|\int_{\tau+}^{R_{+}^{\prime \prime}} H(t, u) d \xi\right| \\
& \leqq \eta_{m} T\left(r_{m}\right)\left\{\left(\frac{R^{\prime \prime}}{r_{m}}\right)^{\mu} H\left(R^{\prime \prime}, u\right)+\left(\frac{\tau}{r_{m}}\right)^{\mu} H(\tau, u)+\int_{\tau}^{R^{\prime \prime}}\left(\frac{t}{r_{m}}\right)^{\mu}\left|\frac{\partial H}{\partial t}\right| d t\right\} .
\end{aligned}
$$

In the latter integral

$$
\frac{\partial H}{\partial t}=-\frac{2 \gamma t^{\gamma-1} u^{\gamma}}{t^{2 \gamma}-u^{2 \gamma}} \quad\left(u \leqq t e^{-\sigma}\right),
$$

and hence, in view of (13.4),

$$
\int_{\tau}^{R^{\prime \prime}}\left(\frac{t}{r_{m}}\right)^{\mu}\left|\frac{\partial H}{\partial t}\right| d t \leqq 2 \gamma r_{m}^{-\mu}\left(1-e^{-2 \sigma \gamma}\right)^{-1} u^{\nu} \int_{u}^{\infty} t^{\mu-\gamma-1} d t \leqq K(\mu, \gamma, s, \sigma),
$$

where $K$ only depends on the parameters explicitly listed.

Use (13.13) in (13.12) and take into account the obvious estimates

$$
\begin{gathered}
0<H\left(R^{\prime \prime}, u\right) \leqq 4\left(\frac{u}{R^{\prime \prime}}\right)^{\gamma} \quad\left(u \leqq \frac{R^{\prime \prime}}{2}\right), \\
0<H(t, u) \leqq \log \frac{1}{\sigma}+\log \left(\frac{2 e^{\gamma}}{\gamma}\right) \quad\left(u \leqq t e^{-\sigma}\right) .
\end{gathered}
$$

Our convention concerning the symbol $\eta_{m}$, leads us to

$$
\left|\int_{\tau+}^{R^{\prime \prime}} H(t, u) d \xi\right| \leqq \eta_{m} T\left(r_{m}\right)
$$

returning to (13.11), we find, 


$$
\Sigma_{2}(u) \leqq \mu B \frac{T\left(r_{m}\right)}{r_{m}^{\mu}} \int_{u}^{\infty} H(t, u) t^{\mu-1} d t+\eta_{m} T\left(r_{m}\right) .
$$

The proof of

$$
\Sigma_{0}(u) \leqq \mu B \frac{T\left(r_{m}\right)}{r_{m}^{\mu}} \int_{0}^{u} H(t, u) t^{\mu-1} d t+\eta_{m} T\left(r_{m}\right)
$$

is entirely analogous and will be left to the reader.

To complete the proof of (13.8), we add (13.14) and (13.15) and observe that

$$
\mu \int_{0}^{\infty} H(t, u) t^{\mu-1} d t=\frac{\mu}{\gamma} u^{\mu} \int_{0}^{\infty} \log \left|\frac{1+t}{1-t}\right| t^{\mu / \gamma-1} d t=\pi u^{\mu} \tan \left(\frac{\pi \mu}{2 \gamma}\right) .
$$

The explicit evaluation of the second integral in (13.16) is straightforward. It may be obtained by residues. We may also expand $\log |(1+t) /(1-t)|$ in positive powers of $t$, for $0 \leqq t<1$, and in negative powers, for $t>1$. Term by term integrations followed by the use of the series of partial fractions which represents the cotangent immediately leads to the closed form in (13.16).

\section{Selection of the points of maximum modulus.}

$\mathrm{L}$ e $\mathrm{m} \mathrm{m} \mathrm{a} \mathrm{14.1.} \mathrm{Let} \mathrm{the} \mathrm{assumptions} \mathrm{of} \mathrm{Lemma} 13.1$ be unchanged. It is then possible to select a positive sequence $\left\{u_{m}\right\}_{m}$ such that

$$
\begin{aligned}
\max _{0 \leqq \theta<2 \pi}\left|f\left(u_{m} e^{i \theta}\right)\right| & =M\left(u_{m}\right)<+\infty, \min _{0 \leqq \theta<2 \pi}\left|f\left(u_{m} e^{i \theta}\right)\right|>0, \\
& \frac{u_{m}}{r_{m}} \rightarrow 1 \quad(m \rightarrow \infty),
\end{aligned}
$$

and

$$
\sum_{R_{m}^{\prime}<\left|b_{j}\right| \leqq R_{m}^{\prime \prime}} H\left(\left|b_{j}\right|, u_{m}\right)=\tilde{Q}_{m} \leqq\left(\pi B \tan \left(\frac{\pi \mu}{2 \gamma}\right)+\eta_{m}\right) T\left(u_{m}\right) \quad\left(\eta_{m} \rightarrow 0\right) .
$$

Proof. Let $\varepsilon(0<\varepsilon<1)$ be given. Choose $\sigma(0<\sigma<1)$ small enough to imply

$$
6 \mu B e^{2 \mu} \sigma\left(K_{4}+\log \frac{1}{\sigma}\right)<\varepsilon \quad(\sigma=\sigma(\varepsilon)),
$$


where $K_{4}>0$ is the constant in (12.11). Hence, by (13.1)

$$
\begin{aligned}
\mathscr{N} & =n\left(e^{2 \sigma} r_{m}\right)-n\left(e^{-\sigma} r_{m}\right)=B T\left(r_{m}\right)\left(e^{2 \sigma \mu}-e^{-\sigma \mu}\right)+\eta_{m} T\left(r_{m}\right) \\
& \leqq\left\{\frac{\varepsilon}{2\left(K_{4}+\log \frac{1}{\sigma}\right)}+\eta_{m}\right\} T\left(r_{m}\right) .
\end{aligned}
$$

We now use Lemma 12.2 to determine in the interval $\left[r_{m}, e^{\sigma} r_{m}\right]$ a point $u_{m}$ such that there are no zeros or poles of $f(z)$ on the circumference $|z|=u_{m}$, and such that

$$
\Sigma_{1}\left(u_{m}\right)=\sum_{r^{e^{-\sigma}}<\left|b_{j}\right| \leqq r_{m} e^{2 \sigma}} H\left(\left|b_{j}\right|, u_{m}\right)<\left(K_{4}+\log \frac{1}{\sigma}\right) \mathscr{N} ;
$$

we then combine (14.5) and (14.6). This leads to

$$
\Sigma_{1}\left(u_{m}\right) \leqq \frac{2}{3} \varepsilon T\left(r_{m}\right) \quad\left(m>m_{0}(\varepsilon)\right) .
$$

From (13.8), (14.6), (14.7) and the definition of $\tilde{Q}_{m}$, we deduce

$$
\widetilde{Q}_{m} \leqq e^{\mu \sigma} T\left(r_{m}\right) \pi B \tan \left(\frac{\pi \mu}{2 \gamma}\right)+\varepsilon T\left(r_{m}\right) \quad\left(m>m_{0}(\varepsilon)\right) .
$$

At this stage, we give to $\varepsilon$ a succession of positive values $\rightarrow 0$; this, in view of (14.4), enables us to select a positive sequence $\left\{\sigma_{m}\right\}$ such that $\sigma_{m} \rightarrow 0$.

Then, by the "diagonalization process" precedingly used, we obtain (14.2) and deduce (14.3) from (14.8). This completes the proof of Lemma 14.1.

15. The maximum modulus of a function satisfying the hypotheses ES.

$\mathrm{L} \mathrm{e} \mathrm{m} \mathrm{m}$ a 15.1. Let $f(z)$ satisfy the hypotheses $E S$, and let $\left\{r_{m}\right\}_{m}$ be the sequence of peaks, of order $\mu$, of $T(r)=T(r, f)$ for which (12) holds.

Put

$$
M(r)=M(r, f)=\max _{\theta}\left|f\left(r e^{i \theta}\right)\right|,
$$

and let $s>0$ be given.

Then

$$
\pi \mu \sin \mu \beta \leqq \liminf _{r \rightarrow \infty} \frac{\log M(r)}{T(r)} \quad(r \in \Lambda(s)) .
$$

Proof. Given $\varepsilon>0$ and $\omega(0<\omega<\pi)$ determine a measurable set $E$ such that

$$
|E|=2 \omega, \quad m^{*}\left(r e^{i \omega}\right)-\varepsilon \leqq \frac{1}{2 \pi} \int_{E} \log \left|f\left(r e^{i \theta}\right)\right| d \theta .
$$


Hence

$$
T^{*}\left(r e^{i \omega}\right)-T^{*}(r)=m^{*}\left(r e^{i \omega}\right) \leqq \frac{\omega}{\pi} \log M(r)
$$

[The above inequality is trivial if $M(r)=+\infty$.]

By (15.2) and Theorem 2,

$$
T(r)(\cos \mu(\beta-\omega)-\cos \mu \beta)-|\eta(r)| T(r) \leqq \frac{\omega}{\pi} \log M(r),
$$

where $\omega$ is fixed $(0<\omega<\beta)$ and $\eta(r) \rightarrow 0$ as $r \rightarrow \infty \quad(r \in \Lambda(s))$.

This leads to

$$
\frac{\pi}{\omega}(\cos \mu(\beta-\omega)-\cos \mu \beta) \leqq \liminf _{r \rightarrow \infty} \frac{\log M(r)}{T(r)} \quad(r \in \Lambda(s)) .
$$

Letting $\omega \rightarrow 0+$, we obtain (15.1).

16. Construction of the sectors $\mathscr{D}_{m}$ and proof of Theorem 3 . We propose to apply Lemma 11.2 taking into account the results of $\S 14$ and $\S 15$. This requires a preliminary definition of the sectors $\mathscr{D}_{m}$ which appear in (11.23).

To construct the sectors $\mathscr{D}_{m}$ :

(i) take $u_{m}, R_{m}^{\prime}, R_{m}^{\prime \prime}$ as in Lemma 14.1;

(ii) for $\omega_{m}$ take any one of the arguments for which

$$
\left|f\left(u_{m} e^{i \omega_{m}}\right)\right|=M\left(u_{m}\right) ;
$$

(iii) define $\gamma$ by (13.3). Since the hypotheses ES are satisfied, the relations (13.4) hold.

(iv) the sectors $\mathscr{D}_{m}$ are obtained by giving to the parameters $R_{m}^{\prime}, R_{m}^{\prime \prime}$, $\omega_{m}, \gamma$, of (11.23), the values which we have introduced above.

We next introduce some auxiliary quantities. Put

$$
\begin{aligned}
L_{m}^{+} & =\gamma^{2} \int_{R_{m}^{\prime}}^{R_{m}^{\prime \prime}} m\left(t, f ;\left[\omega_{m}-\beta, \omega_{m}+\beta\right]\right) X\left(t, u_{m}\right) \frac{d t}{t} \\
& =\gamma^{2} \int_{R_{m}^{\prime}}^{R_{m}^{\prime \prime}} m(t, f) X\left(t, u_{m}\right) \frac{d t}{t}-\tilde{L}_{m}
\end{aligned}
$$

where 


$$
\tilde{L}_{m}=\gamma^{2} \int_{R_{m}^{\prime}}^{R_{m}^{\prime \prime}} m\left(t, f ;\left[\omega_{m}+\beta, 2 \pi+\omega_{m}-\beta\right]\right) X\left(t, u_{m}\right) \frac{d t}{t} .
$$

We also need

$$
\begin{aligned}
L_{m}^{-}=\gamma^{2} \int_{R_{m}^{\prime}}^{R_{m}^{\prime \prime}} m\left(t, \frac{1}{f} ;\left[\omega_{m}-\beta, \omega_{m}+\beta\right]\right) X\left(t, u_{m}\right) \frac{d t}{t}, \\
\mathscr{Z}_{m}=\sum_{a_{j} \in \mathscr{D}_{m}} H\left(a_{j} e^{-i \omega_{m}}, u_{m}\right), \\
\mathscr{P}_{m}=\sum_{b_{j} \in \mathscr{D}_{m}} H\left(b_{j} e^{-i \omega_{m}}, u_{m}\right) .
\end{aligned}
$$

All the quantities $L, \mathscr{Z}, \mathscr{P}$ are clearly nonnegative. Let

$$
a=|a| e^{i \varphi}, \quad b=|b| e^{i \varphi},
$$

denote, respectively, a zero and a pole of $f(z)$.

Given $s>0$ and $\eta(0<\eta<\beta)$, consider all those poles for which

$$
e^{-s} u_{m}<|b| \leqq e^{s} u_{m}
$$

let, there be exactly $v_{m}=v_{m}(s)$ of them. We subdivide these poles into three collections $\mathscr{C}_{m}^{(j)}(s, \eta)(j=1,2,3)$ containing, respectively $v_{m}^{(j)}(s, \eta)$ elements and characterized as follows:

(i) the elements of $\mathscr{C}_{m}^{(1)}$ are those for which $\left|\varphi-\omega_{m}\right|<\eta$;

(ii) the elements of $\mathscr{C}_{m}^{(2)}$ are those for which $\eta \leqq\left|\varphi-\omega_{m}\right| \leqq \beta$;

(iii) the elements of $\mathscr{C}_{m}^{(3)}$ are those for which $\beta<\left|\varphi-\omega_{m}\right| \leqq \pi$.

With the notations of Theorem 3, we have

$$
\mu_{m}=v_{m}^{(2)}+v_{m}^{(3)} \text {. }
$$

Notice that Lemma 12.1 and (16.7) yield

$$
\sum_{b_{j} \in \mathscr{C}_{m}^{(2)}}\left\{H\left(\left|b_{j}\right|, u_{m}\right)-H\left(b_{j} e^{-i \omega_{m}}, u_{m}\right)\right\} \geqq K_{5}(s, \gamma, \eta) v_{m}^{(2)}
$$

the constant $K_{5}>0$, only depends on the parameters explicitly listed.

For all poles satisfying (16.7) we have, by (12.6) (with $\delta=\pi, \psi=0$ ),

$$
H\left(\left|b_{j}\right|, u_{m}\right) \geqq e^{s \gamma}\left(1+e^{s \gamma}\right)^{-2}=K_{6}(s, \gamma) .
$$

Hence, 


$$
\sum_{b_{j} \in \mathscr{C}_{m}^{(3)}} H\left(\left|b_{j}\right|, u_{m}\right) \geqq v_{m}^{(3)} K_{6} .
$$

Similarly consider the zeros of $f(z)$ characterized by

$$
u_{m} e^{-s}<|a| \leqq e^{s} u_{m}, \quad\left|\psi-\omega_{m}\right| \leqq \beta-\eta=\frac{\pi-\eta \gamma}{\gamma} ;
$$

with the notations of Theorem 3 there are $x_{m}=x_{m}(s, \eta)$ of them.

By (16.5), (12.5) and (12.6)

$$
\mathscr{Z}_{m} \geqq x_{m} K_{7}(s, \gamma, \eta) .
$$

In order to apply Lemma 11.2 we still need to estimate

$$
L_{m}^{+}+\tilde{L}_{m}=\gamma^{2} \int_{R_{m}^{\prime}}^{R_{m}^{\prime \prime}}\{T(t)-N(t, f)\} X\left(t, u_{m}\right) \frac{d t}{t}
$$

By (15), (16) and (14.2)

$$
\begin{aligned}
T(t) & -N(t, f) \leqq(1+\eta(t))(1-\cos \beta \mu) T(t) \\
& \leqq\left(1+\eta_{m}\right)(1-\cos \beta \mu) T\left(u_{m}\right)\left(\frac{t}{u_{m}}\right)^{\mu} \quad\left(R_{m}^{\prime} \leqq t \leqq R_{m}^{\prime \prime}\right) .
\end{aligned}
$$

Hence, combining (16.12), (16.13) and the change of variable $\left(t / u_{m}\right)^{\gamma}=x$, we obtain

$$
\begin{aligned}
L_{m}^{+}+\tilde{L}_{m} & \leqq\left(1+\eta_{m}\right)(1-\cos \beta \mu) T\left(u_{m}\right) \gamma \int_{0}^{\infty} \frac{x^{\mu / \gamma}}{(1+x)^{2}} d x \\
& =\left(1+\eta_{m}\right)(1-\cos \beta \mu) T\left(u_{m}\right) \frac{\pi \mu}{\sin \beta \mu} .
\end{aligned}
$$

The application of Lemma 11.2 is now immediate. Using (16.1), (16.2), (16.4), (16.5) and (16.6) in (11.24) we first deduce

$$
\log M\left(u_{m}\right)+\mathscr{Z}_{m}+L_{m}^{-}=L_{m}^{+}+\mathscr{P}_{m}+\eta_{m} T\left(u_{m}\right),
$$

and remark that

$$
\begin{aligned}
\mathscr{P}_{m} \leqq & \sum_{b_{j} \in\left\{\mathscr{C}_{m}^{(1)} \cup \mathscr{C}_{m}^{(2)}\right\}} H\left(b_{j} e^{-i \omega_{m}}, u_{m}\right) \\
& +\sum_{R_{m}^{\prime}<\left|b_{j}\right| \leqq e^{-s_{u_{m}}}} H\left(\left|b_{j}\right|, u_{m}\right)+\sum_{e^{s_{u_{m}}<\left|b_{j}\right| \leqq R_{m}^{\prime \prime}}} H\left(\left|b_{j}\right|, u_{m}\right) .
\end{aligned}
$$


Hence (12.3), (16.9) and (16.10) yield

$$
\mathscr{P}_{m}+v_{m}^{(2)} K_{5}+v_{m}^{(3)} K_{6} \leqq \sum_{R_{m}^{\prime}<\left|b_{j}\right| \leqq R_{m}^{\prime \prime}} H\left(\left|b_{j}\right|, u_{m}\right)
$$

and in view of (14.3) and (16.8)

$\mathscr{P}_{m}+\mu_{m} K_{8} \leqq\left(\pi \mu \cos \beta \mu \tan \frac{\beta \mu}{2}+\eta_{m}\right) T\left(u_{m}\right) \quad\left(K_{8}=\min \left(K_{5}, K_{6}\right)\right)$.

Now use in (16.15), the relations (16.11), (16.14) and (16.16). This leads to

$$
\begin{aligned}
& \log M\left(u_{m}\right)+x_{m} K_{7}+\mu_{m} K_{8}+L_{m}^{-}+\tilde{L}_{m} \\
& \leqq \pi \mu T\left(u_{m}\right)\left(\frac{1-\cos \beta \mu}{\sin \beta \mu}+\cos \beta \mu \tan \frac{\beta \mu}{2}\right)+\eta_{m} T\left(u_{m}\right) \\
& =\left(\pi \mu \sin \beta \mu+\eta_{m}\right) T\left(u_{m}\right),
\end{aligned}
$$

and consequently

$$
\limsup _{m \rightarrow \infty} \frac{\log M\left(u_{m}\right)}{T\left(u_{m}\right)} \leqq \pi \mu \sin \beta \mu .
$$

Comparing (16.18) and (15.1) we obtain assertion IV of Theorem 3.

Returning to (16.17) we establish (26) as well as the following

L e m m a 16.1. Let $f(z)$ satisfy the hypotheses $E S$. Then, as $m \rightarrow \infty$,

$$
L_{m}^{-}=o\left(T\left(u_{m}\right)\right), \quad \tilde{L}_{m}=o\left(T\left(u_{m}\right)\right) .
$$

The proof of Theorem 3 is now complete.

The additional information contained in Lemma 16.1 is of some importance. In a continuation of this paper we propose to show that Lemma 16.1 leads to a complete description of the asymptotic behavior of $f(z)$ throughout annuli such as

$$
e^{-s} u_{m} \leqq|z| \leqq e^{s} u_{m} \quad(s>0, m=1,2,3, \ldots) .
$$

\section{References}

[1] BAERnstein, A., II: Proof of Edrei's spread conjecture. - Proc. London Math. Soc. (3) 26, 1973, 418-434.

[2] EDREI, A.: Sums of deficiencies of meromorphic functions. II. - J. Analyse Math. 19, 1967, 53-74.

[3] -"- Locally tauberian theorems for meromorphic functions of lower order less than one. - Trans. Amer. Math. Soc. 140, 1969, 309-332. 
[4] EDREI, A.: A local form of the Phragmén-Lindelöf indicator. - Mathematika $17,1970,149-172$.

[5] EDREI, A., and W. H. J. Fuchs: Bounds for the number of deficient values of certain classes of meromorphic functions. - Proc. London Math. Soc. (3) $12,1962,315-344$.

[6] Fuchs, W. H. J.: Topics in Nevanlinna theory. - Proceedings of the NRL conference on classical function theory, Mathematics Research Center, Naval Research Laboratory, Washington, D.C., 1970, 1-32.

[7] Hardy, G. H., and W. W. Rogosinski: Notes on Fourier series. III. Asymptotic formulae for the sums of certain trigonometrical series. - Quart. J. Math. Oxford Ser. 16, 1945, 49-58.

[8] LiTTLEWOOD, J. E.: Lectures on the theory of functions. - Oxford University Press, London etc., 1944.

[9] Petrefnko, V.P. [В. П. Петренко]: Рост мероморфных функций конечного нижего порядка. - Izv. Akad. Nauk SSSR Ser. Mat. 33, 1969, 414-454. Translation: Growth of meromorphic functions of finite lower order. Math. USSR - Izv. 3, 1969, 391-432.

[10] TiTchmarsh, E. C.: The theory of functions. - [2nd edition.] Oxford University Press, London, 1939.

Syracuse University

Department of Mathematics

Syracuse, New York 13210

USA

Received 25 August 1975

\author{
Cornell University \\ Department of Mathematics \\ Ithaca, New York 14850 \\ USA
}

